\title{
An Offset Laser Measurement Method for the Deviation Analysis of Cylindrical Gear
}

Ning Mei ( $\sim$ lanbin1997@126.com )

Yangzhou University

Aiping Song

School of Mechanical Engineering, Yangzhou University, Yangzhou225127, People's Republic of China

Chenwei Yu

School of Mechanical Engineering, Yangzhou University, Yangzhou225127, People's Republic of China Jianzhou Pan

School of Mechanical Engineering, Yangzhou University, Yangzhou225127, People's Republic of China

\section{Research Article}

Keywords: Laser displacement sensor, Gear measurement, Offset distance, Gear deviation

Posted Date: March 17th, 2021

DOl: https://doi.org/10.21203/rs.3.rs-306651/v1

License: (c) (1) This work is licensed under a Creative Commons Attribution 4.0 International License.

Read Full License 


\title{
An offset laser measurement method for the deviation analysis of cylindrical gear
}

\author{
Ning Mei, Aiping Song, Chenwei Yu, Jianzhou Pan \\ School of Mechanical Engineering, Yangzhou University, Yangzhou225127, People's Republic of China \\ Corresponding author: Aiping Song \\ Email: songaiping@163.com
}

\begin{abstract}
Generally, in the laser measurement of gears, the laser beam passes through the center of the gear, and the laser displacement sensor reads the spatial distance from the gear involute tooth surface to the laser displacement sensor. However, in this method, the angle between the laser beam and the normal vector of the measured tooth surface is too large, which affects the accuracy of the measurement and the stability of the data. This paper proposes an offset laser measurement method. The laser beam is offset from the center of the gear by a certain distance to form a larger incident angle with the tooth surface, which can effectively improve the problem and increase the measurement accuracy. Through the discussion of selecting the optimal offset distance, the range of optimal offset measurement position is obtained and clarified by experiments. We solved the data conversion problem caused by the change of measuring position, and measured the pitch deviation and helix angle of the gear to confirm the feasibility of this method. Based on theoretical calculation and experimental verification, it is found that this method has the advantages of better measurement accuracy and less measurement data fluctuations. It is suitable for precision gear measurement.
\end{abstract}

Keywords: Laser displacement sensor, Gear measurement, Offset distance, Gear deviation

\section{Introduction}

Gear measurement plays an important role in the quality assessment of finished products, and it is also a technical guarantee for quality control during processing. With the development of industrial technology and the increasing requirements for gears, comprehensive gear measurement has begun to be widely used. The mainstream gear measuring equipment mainly includes gear meshing tester, $\mathrm{CNC}$ gear measuring center, on-line measuring and sorting machine for gears, laser measuring instrument, 
etc. [1]. The gear meshing tester is to mesh and rotate the measured gear with the ideal and accurate measuring gear, and the recorded error curve can reflect the multiple errors of the gear. It is mainly divided into single-sided meshing and double-sided meshing comprehensive measuring instruments. The single-sided meshing comprehensive measuring instrument is divided into mechanical type, grating type, magnetic index type and inertial type, and its movement is close to the state of gear in use [2]. The gear measuring center is similar in principle to the polar coordinate measuring machine. The probe produces the required measurement motion relative to the workpiece, and the computer collects the indication value of the micrometer and the actual position of each coordinate axis to obtain the measurement result. This method has high precision but large amount of data calculation and long measurement time.

In recent years, laser displacement sensors have many applications in the detection of gear geometric accuracy and transmission stability. Ma et al. [3] proposed an experimental device composed of a special-fabricated micro-displacement platform and a pair of laser displacement sensors to measure the vertical and horizontal displacement of the flexspline of harmonic drive gears to determine the radial displacement. Tian et al. [4] proposed using two opposing adjustable laser sensors to obtain tooth profile information through one scan, and reconstruct the key geometric model of the gear profile after compensation. Raghuwanshi et al. [5] presented a laser displacement sensor technique to measure the tooth deflection of spur gear to calculate the friction stiffness of cracked teeth, but the experimental device for the determination of tooth surface friction is not very clear. Jiang et al. [6] found the method of use laser displacement sensor to measure the bending deformation and stiffness of the output shaft to obtain the friction force. Qiu[7] proposed a non-contact gear measurement method to measure various geometric errors of gears by building a gear detection platform. However, as described in [8,9], if the laser beam passes through the center of the gear during measurement, the angle between the laser beam and the normal vector of the tooth surface is large to form an irregular elliptical spot, which affects the detection accuracy. Pei et al. [10] optimized the installation angle and position of the laser displacement sensor to improve the problem, but during the measurement process, the previous tooth blocks the measuring laser beam of the latter tooth in a 
large range, which leads to a decrease in the effective data of the measurement.

Therefore, this paper proposes an offset laser measurement method for the deviation analysis of cylindrical gear to improve these problems and increase the accuracy of the measurement. Firstly, a mathematical model of the best offset selection is established to clarify the selection range of the offset, and the best measurement position is selected according to the offset value and the range of the laser displacement sensor. Then, develop offset measurement control software to control the three-coordinate translation device and the workpiece turntable, and record the values of the laser displacement sensor and the encoder on the turntable during the measurement process to obtain the measurement data of the tooth profile. Finally, the various parameters and deviations of the gear are obtained by coordinate conversion and data analysis of the measurement data.

\section{The influence of spot shape on laser measurement}

The laser displacement sensor used in gear measurement is based on triangulation. As shown in Fig.1, the laser beam emitted by the laser source hits $H_{1}$ on the surface of the object, $H_{0}$ is a point on the reference plane, and the imaging of the two points on the CCD is $M_{1}$ and $M_{0}$. Draw the perpendicular from $H_{1}, M_{1}$ to $H_{0} M_{0}$ to get $B, D$. The triangle $M_{1} D O$ is similar to the triangle $H_{1} B O$. The distance of $B H_{1}$ can be calculated according to the triangle similarity rule, and then the distance of $y$ can be obtained. 


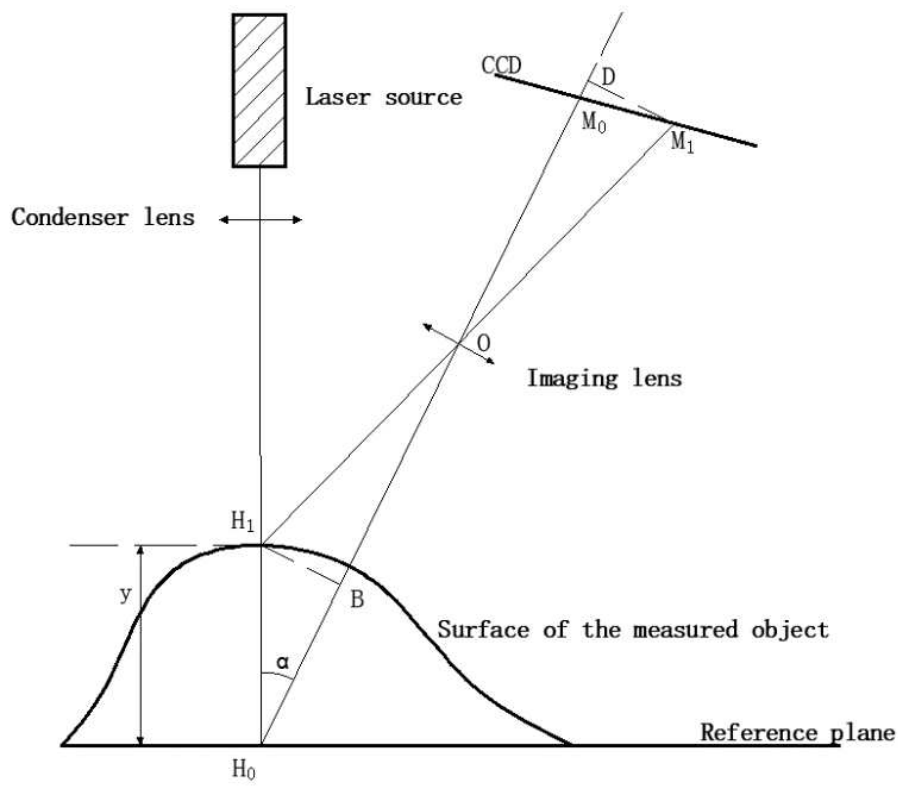

Fig. 1 The principle of triangulation

According to the principle of triangulation, the calculation of length $y$ is restricted by the accuracy of the two points $M_{0} M_{1}$, while the accuracy of the two points is affected by the shape and size of the spot. The tooth surface is a complex space curve, and its curvature changes constantly, which easily leads to measurement fluctuations. Huang [11] proposed an object surface inclination error. It is obtained through experiments that when the tilt angle is within $5^{\circ}$, the measurement error can be controlled at $0.12 \mathrm{~mm}$, and when the tilt angle is $30^{\circ}$, the maximum measured displacement error has reached $0.5 \mathrm{~mm}$.

When the measured surface is close to a flat surface, the laser beam hits the surface with a uniform circular spot, which is mainly common in the measurement of gear addendum and dedendum. At this time, the angle between the normal vector of the tooth profile and the laser beam is close to $0^{\circ}$, Which is equivalent to the laser beam hitting the surface vertically. At this time, it has no effect on the accuracy of the laser beam. When the curvature of the surface changes continuously and there is a large height difference, the laser beam hits the surface with an uneven and approximate ellipse. At this time, the angle between the normal vector of the tooth profile and the laser beam is close to $90^{\circ}$, which is equivalent to the laser beam is tangent to the surface. The nonlinear error in the measurement system increases, which is mainly common in the measurement of the tooth profile. Therefore, by 
improving the angle between laser beam and tooth profile and making the maximum angle within a reasonable range, the accuracy of tooth profile measurement can be greatly improved. Fig.2 and Fig. 3 are schematic diagrams of the laser beam spots when the laser beam is at different positions on the cylinder shaft. Fig. 2 is the spot formed by the laser beam directly facing the center of the shaft. The spot is uniform and convergent. Fig.3 shows the spot formed by the laser beam on the steep surface of the cylinder shaft, which is uneven and has large scattering during practical application.
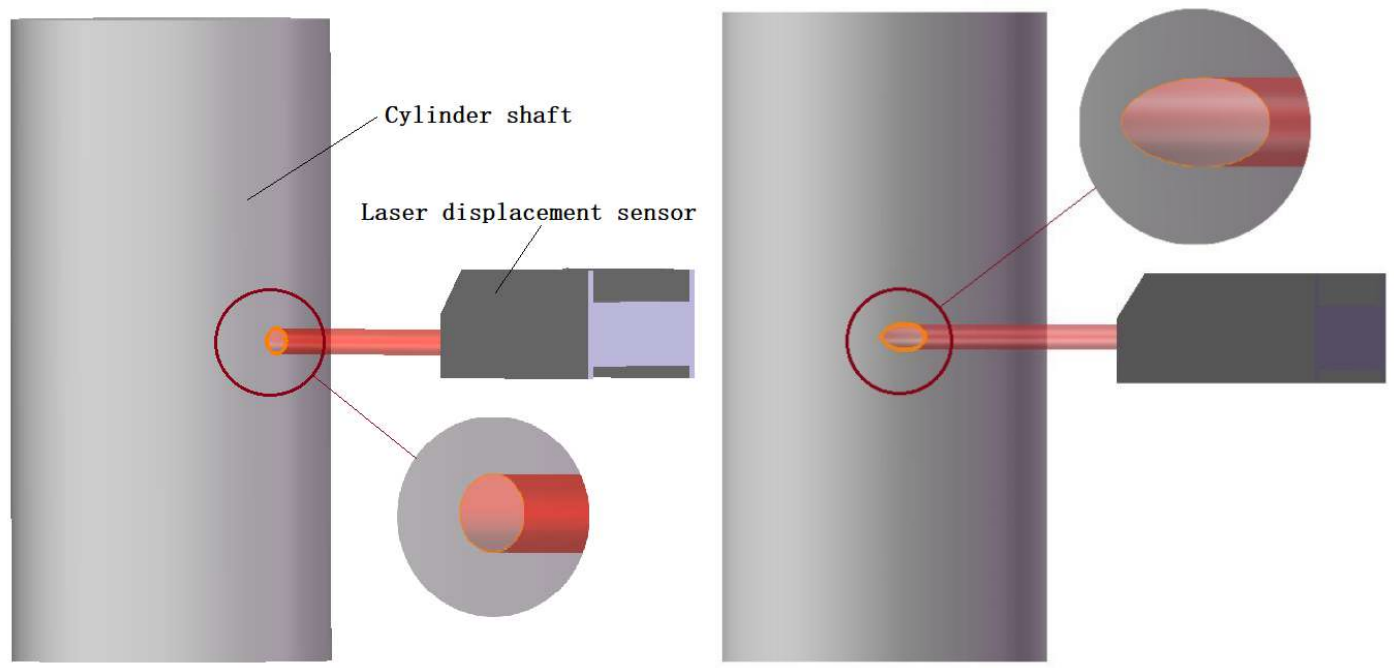

Fig. 2 The spot shape when laser beam facing the center of the shaft Fig. 3The spot shape when laser beamoff-center

As shown in Fig.4, $O_{b}$ is the center of the base circle, $H\left(x_{H}, y_{H}\right)$ is the measured point on the gear, $L_{1}$ is the line connection the center of the base circle and point $H$, and $L_{2}$ is the normal line of the involute at point $H$, the radius of the base circle is $r_{b}$. After the involute has turned a certain angle the center of the base circle is marked as $O_{b}\left(-x_{0},-y_{0}\right)$, and $\alpha_{H}$ is the pressure angle at point $H, \mathrm{k}$ is the slope of $L_{2}$, the line $L_{2}$ where the normal vector of the tooth profile at point $H$ can be expressed as: 


$$
\left\{\begin{array}{l}
y=k\left(x-x_{H}\right)+y_{H} \\
k=\tan \left(\arctan \left(\frac{y_{H}+y_{0}}{x_{H}+x_{0}}\right)+\frac{\pi}{2}-\alpha_{H}\right) \\
\alpha_{H}=\frac{r_{b}}{O_{b} H}
\end{array}\right.
$$

During offset measurement, the laser beam is offset by a distance $e$ along the x-axis. If the angle between the normal of the tooth profile and the x-axis is $\varphi$, the angle between the normal of the tooth profile and the laser beam is $\frac{\pi}{2}-\varphi$. It can be seen that the larger the $\mathrm{k}$ value is, the larger the $\varphi$ value is, the smaller the angle $\frac{\pi}{2}-\varphi$ is, and the more stable the measured value is. On the contrary, the smaller the $\mathrm{k}$ value is, the smaller the $\varphi$ value is, the larger the angle $\frac{\pi}{2}-\varphi$ is, and the fluctuation of measurement data increases.

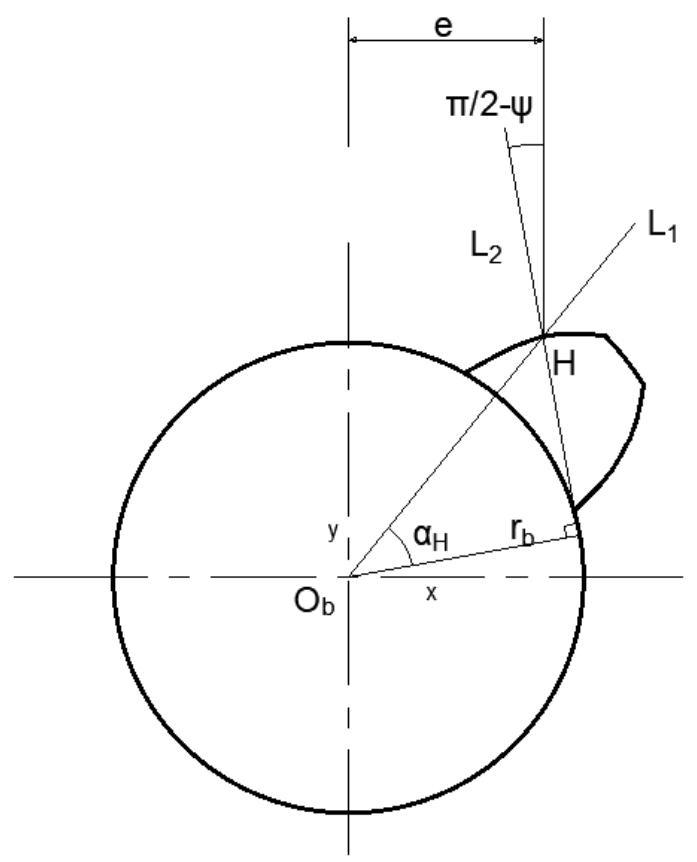

Fig. 4 Schematic diagram of the normal of the tooth profile

\section{Principle of offset laser measurement method}


During measurement, the laser displacement sensor measures the distance from the laser emitting point to the tooth surface, and the encoder feeds back angle information. When the laser passes through the center of the gear, the sensor and the encoder reflect the position and angle data of the same point. In the offset laser measurement, the y-axis of the measurement coordinate system and the workpiece coordinate system are not collinear. The position and angle data actually correspond to the position data of the measured point and the gear rotation angle. The corresponding position and angle of the current point can be obtained through coordinate system conversion.

The schematic diagram of offset measurement data conversion is shown in Fig. 5. The offset distance between the laser displacement sensor and the gear center is $e, l_{d}$ is the distance from the sensor to the measured point, the distance from the sensor to the $\mathrm{X}$ axis is $d$, and the distance from the measured point to the $\mathrm{X}$ axis is $l=d-l_{d}$. Take any two points $p_{m}$ and $p_{n}$ on the gear, and the measured data of the two points are $p_{m}\left(l_{d m}, \theta_{m}\right), p_{n}\left(l_{d n}, \theta_{n}\right)$. Taking $p_{m}$ as the initial point, the angle between $p_{m}$ and the $\mathrm{X}$ axis is $\operatorname{arcos}\left(e / r_{m}\right)$, the angle rotated by the gear from $p_{m}$ to $p_{n}$ is $\theta_{n}-\theta_{m}$, and the angle between $p_{n}$ and the $\mathrm{Y}$ axis is $\operatorname{arsin}\left(e / r_{n}\right)$, then the angle between $p_{m}$ and $p_{n}$ relative to the gear coordinate system is:

$$
\theta=\operatorname{arcos}\left(e / r_{m}\right)+\theta_{n}-\theta_{m}+\operatorname{arsin}\left(e / r_{n}\right)-\pi / 2
$$

The measurement result can be transformed into a polar coordinate formula:

$$
\left\{\begin{array}{l}
r=\sqrt{e^{2}+l^{2}}=\sqrt{e^{2}+\left(\mathrm{d}-l_{d}\right)} \\
\theta=\operatorname{arcos}\left(e / r_{m}\right)+\theta_{n}-\theta_{m}+\operatorname{arsin}\left(e / r_{n}\right)-\pi / 2
\end{array}\right.
$$

When taking consecutive points for iterative calculation:

$$
\theta_{n}=\Delta \theta+\theta_{n-1}=\operatorname{arcos}\left(e / r_{n-1}\right)+\theta_{n}+\operatorname{arsin}\left(e / r_{n}\right)-\pi / 2
$$

Then the conversion formula becomes:

$$
\left\{\begin{array}{l}
r_{n}=\sqrt{e^{2}+l^{2}}=\sqrt{e^{2}+\left(\mathrm{d}-l_{d}\right)} \\
\theta_{n}=\operatorname{arcos}\left(e / r_{n-1}\right)+\theta_{n}+\operatorname{arsin}\left(e / r_{n}\right)-\pi / 2
\end{array}\right.
$$




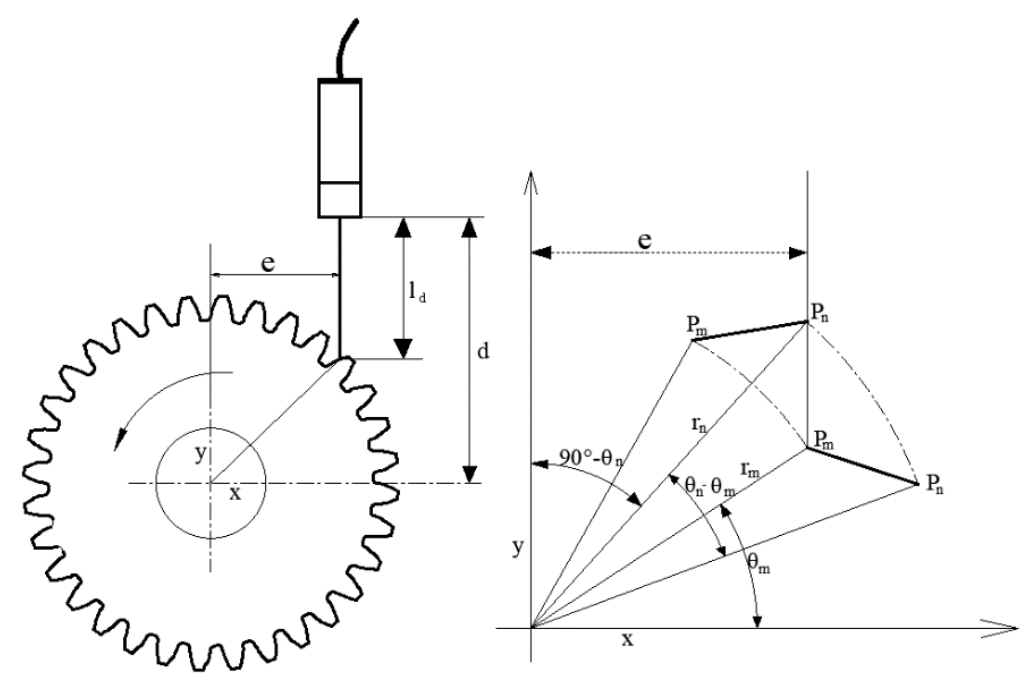

Fig. 5 Schematic diagram of offset laser measurement method

\section{Basis for offset selection}

The offset selection is affected by many factors. The offset value is small, and the problem of laser incident angle is not improved. The larger the offset value is, the more the previous tooth cover the latter tooth, and effective data of the measurement is reduced, which cannot accurately reflect the gear error. In the selection of the offset, the front tooth occlusion degree $A_{M}$, the slope of the tooth profile normal line k, and the overall data efficiency $D_{M}$ are introduced to discuss the best selection range of the offset.

The tooth number is $\mathrm{z}$, the modulus is $m$, the addendum radius is $r_{a}$, the dedendum radius is $r_{f}$, the reference circle radius is $r$, the tooth thickness at the reference circle is $\mathrm{s}$, and the pressure angle is $\alpha . \alpha_{M}$ is the angle occupied by the effective part of the measured point on the tooth profile, $\alpha_{\text {overall }}$ is the gear center angle occupied by the single side tooth line, $s_{a}=\frac{s r_{a}}{r}-2 r_{a}\left(\right.$ inv $\left.\alpha_{a}-i n v \alpha\right)$ is the gear center angle occupied by the addendum, and $s_{f}=\frac{s r_{f}}{r}-2 r_{f}\left(i n v \alpha_{f}-i n v \alpha\right)$ is the gear center angle occupied by the dedendum, the front tooth occlusion degree $A_{M}$ can be expressed as:

$$
A_{M}=\frac{\alpha_{M}}{\alpha_{\text {overall }}}, \text { where } \alpha_{\text {overall }}=\frac{\pi}{z}-\frac{s_{a}}{2}-\frac{s_{f}}{2} \text {. }
$$


When considering the angle between the laser beam and the tooth profile normal, the angle can be judged according to the slope of the tooth profile normal line $\mathrm{k}$. The center of the basic circle is $O_{b}(0,0)$, and after the involute turns a certain angle, it is recorded as $O_{b}\left(-x_{0},-y_{0}\right), H\left(x_{H}, y_{H}\right)$ is the measured point, the basic circle radius is $r_{b}$, and $\alpha_{H}$ is the pressure angle at point $H$, then the normal line of the tooth profile tooth profile is: $\left\{\begin{array}{l}y=k\left(x-x_{H}\right)+y_{H} \\ k=\tan \left(\arctan \left(\frac{y_{H}+y_{0}}{x_{H}+x_{0}}\right)+\frac{\pi}{2}-\alpha_{H}\right) \text {. As the value } \\ \alpha_{k}=\frac{r_{b}}{O_{b} H}\end{array}\right.$ of $\mathrm{k}$ decreases, the angle between the normal of the tooth profile and the laser beam increases, and the fluctuation of the measurement data increases. In order to improve the angle during the measurement process, the angle should be close to parallel, that is, the maximum $\mathrm{k}$ on the involute line should be kept at a large value under the offset. To make the angle less than $30^{\circ}$, the absolute value of $\mathrm{k}$ should be larger than $\sqrt{3}$.

The rationality of the offset value selection can be judged by the overall data efficiency. $D$ is the number of valid data points in the measurement result data, $D_{\text {overall }}$ is always all the data points of the measurement result, the overall data efficiency can be expressed as:

$$
D_{M}=\frac{D}{D_{\text {overall }}}
$$

Combining the three factors, the best offset value can be taken as:

$\operatorname{MAX} \quad\left\{A_{M} \cdot K \cdot \mathrm{D}_{M}\right\}$

Boundary conditions: $\left\{\begin{array}{l}A_{M} \geq 1 \\ \sqrt{3} \geq \text { Kor } K \leq-\sqrt{3}\end{array}\right.$

For example, an involute gear with a number of teeth of 20 , a modulus of 3 , and a pressure angle of $20^{\circ}$, assuming no fluctuations in the measurement data, then $D=1 . H\left(x_{H}, y_{H}\right)$ is a point on the 


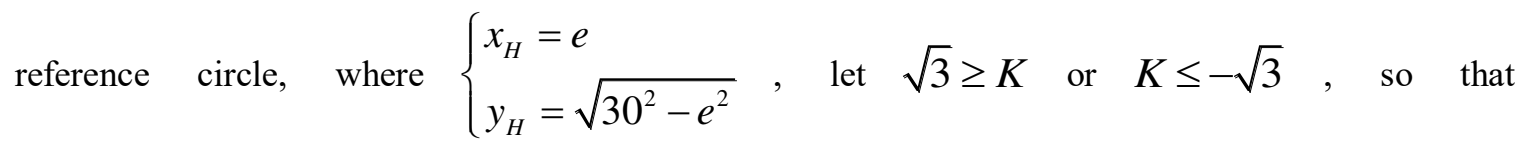
$\frac{\pi}{3}<\arctan \left(\frac{y_{H}+y_{0}}{x_{H}+x_{0}}\right)+\frac{\pi}{2}-\alpha_{H}<\frac{2 \pi}{3}$, the solution is $e \geq 15 . A\left(x_{a}, y_{a}\right)$ is a point on the addendum, make the point of the addendum just cover the dedendum, and the angle between the addendum and the dedendumt is $\theta=\frac{2 \pi}{z}-s_{a}$, where $\left\{\begin{array}{l}x_{a}=e \\ y_{a}=\sqrt{33^{2}-e^{2}}=r_{a} \cos \alpha\end{array}\right.$, $\alpha=\arcsin \left(\frac{r_{f}}{r_{a}^{2}+r_{f}^{2}-2 r_{a} r_{f} \cos \theta} \sin \theta\right)$, let $A_{M} \geq 1$, the solution is $e \leq 21.54$. The value of the offset is between 15 and 21.54 .

\section{Deviation analysis method}

\subsection{Pitch deviation analysis method}

After data conversion, the measurement data has changed from offset measurement data to gear center measurement data. The unfolding angle of any data point on the gear coordinate system is:

$$
\beta_{i}=\left(\mathrm{Y}_{i}-\mathrm{Y}_{1}\right) \cdot \frac{\alpha}{Y_{n}-\mathrm{Y}_{1}}
$$

Where, $Y_{i}$ is the number of pulses corresponding to any point, $Y_{1}$ Is the number of pulses at the first point, $\mathrm{Y}_{n}$ Is the number of pulses at the last point

The data points are expanded according to the polar coordinate data of the gear, and the coordinates of the intersection point $P_{i}$ of the fitted tooth profile and the theoretical reference circle are determined. Using the dichotomy iteration to find points near the reference circle, the pitch value of the interpolation curve of each tooth profile is:

$$
P_{I-1} P_{i}=r_{m} \cdot 2 \arcsin \left(\frac{\sqrt{\left(y_{i}-y_{i-1}\right)^{2}+\left(x_{i}-x_{i-1}\right)^{2}}}{2 r_{m}}\right)
$$

Where, $r_{m}$ is the reference circle radius, $\left(x_{i}, y_{i}\right)\left(x_{i-1}, y_{i-1}\right)$ are the coordinate data of the interpolation point after iteration. 


\subsection{Helix angle analysis method}

Figure 6 is the schematic diagram of helix angle analysis. Suppose the fitted line equation is $z=b_{0}+b_{1} y$, the sum of squared deviations from 3 points to the line is $\sum_{\mathrm{i}=1}^{3} \delta_{i}^{2}=\left[\mathrm{z}_{i}-\left(\mathrm{b}_{0}+\mathrm{b}_{1} \mathrm{y}_{i}\right)\right]^{2}$ using the least square method, the derivative of $b_{i}$ is obtained:

$$
\left[\begin{array}{cc}
3 & \sum_{i=1}^{3} y_{i} \\
\sum_{i=1}^{3} y_{i} & \sum_{i=1}^{3} y_{i}^{2}
\end{array}\right]\left[\begin{array}{l}
b_{0} \\
b_{1}
\end{array}\right]=\left[\begin{array}{l}
\sum_{i=1}^{3} z_{i} \\
\sum_{i=1}^{3} z_{i} y_{i}
\end{array}\right] \text {, where } \beta=\arctan b_{1} \text { 。 }
$$

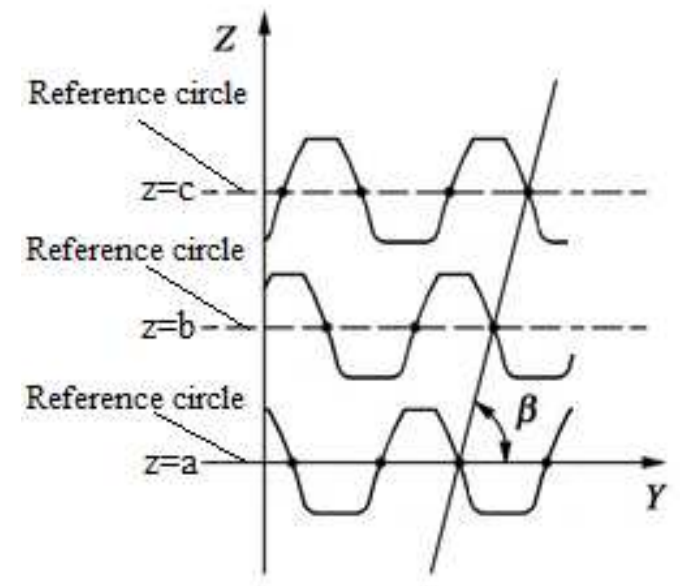

Fig. 6 Schematic diagram of helix angle analysis

\section{Measuring device and software system}

As shown in Fig. 7, the designed measuring device is mainly composed of three parts, the computer control system, the servo system and the grating feedback system. The position data is measured by the laser displacement sensor. The angle data is read by the encoder on the workpiece turntable. The spatial position and movement of the laser displacement sensor are fed back by the grating of three coordinates. 


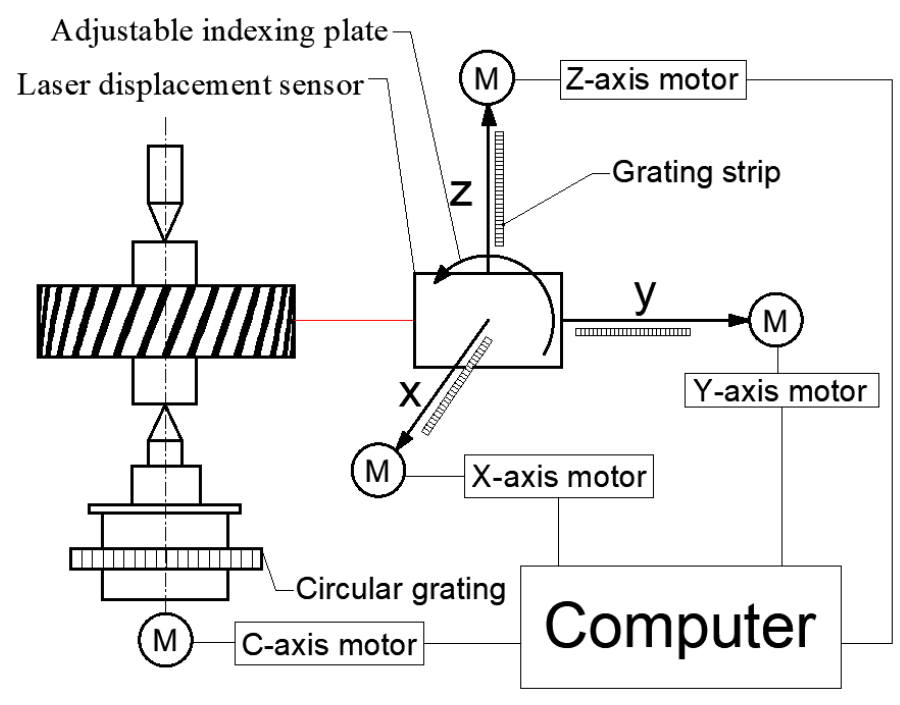

Fig.7 Measurement system

As shown in Fig. 8. The device is a vertical structure. The three-coordinate displacement device is equipped with a laser displacement sensor, which can move linearly in the $\mathrm{X}, \mathrm{Y}$, and $\mathrm{Z}$ directions. The laser displacement sensor and the guide rail are connected by an adjustable indexing plate, which can be adjusted on the YOZ plane. The workpiece turntable is driven by a motor with a built-in encoder. The spatial position of the laser beam and gear is calibrated by the reference shaft. Parts with holes can be installed on the shaft for measurement. 


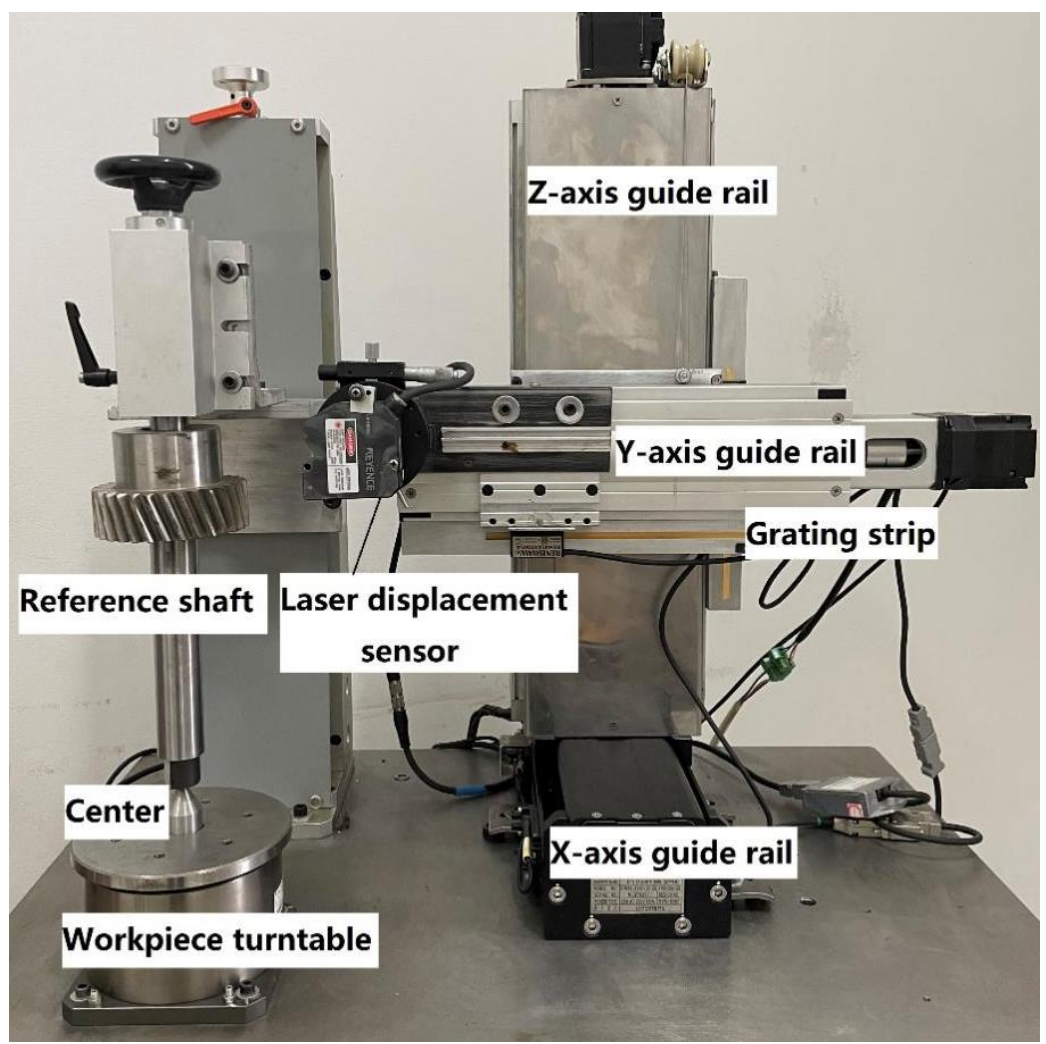

Fig.8 The measurement device

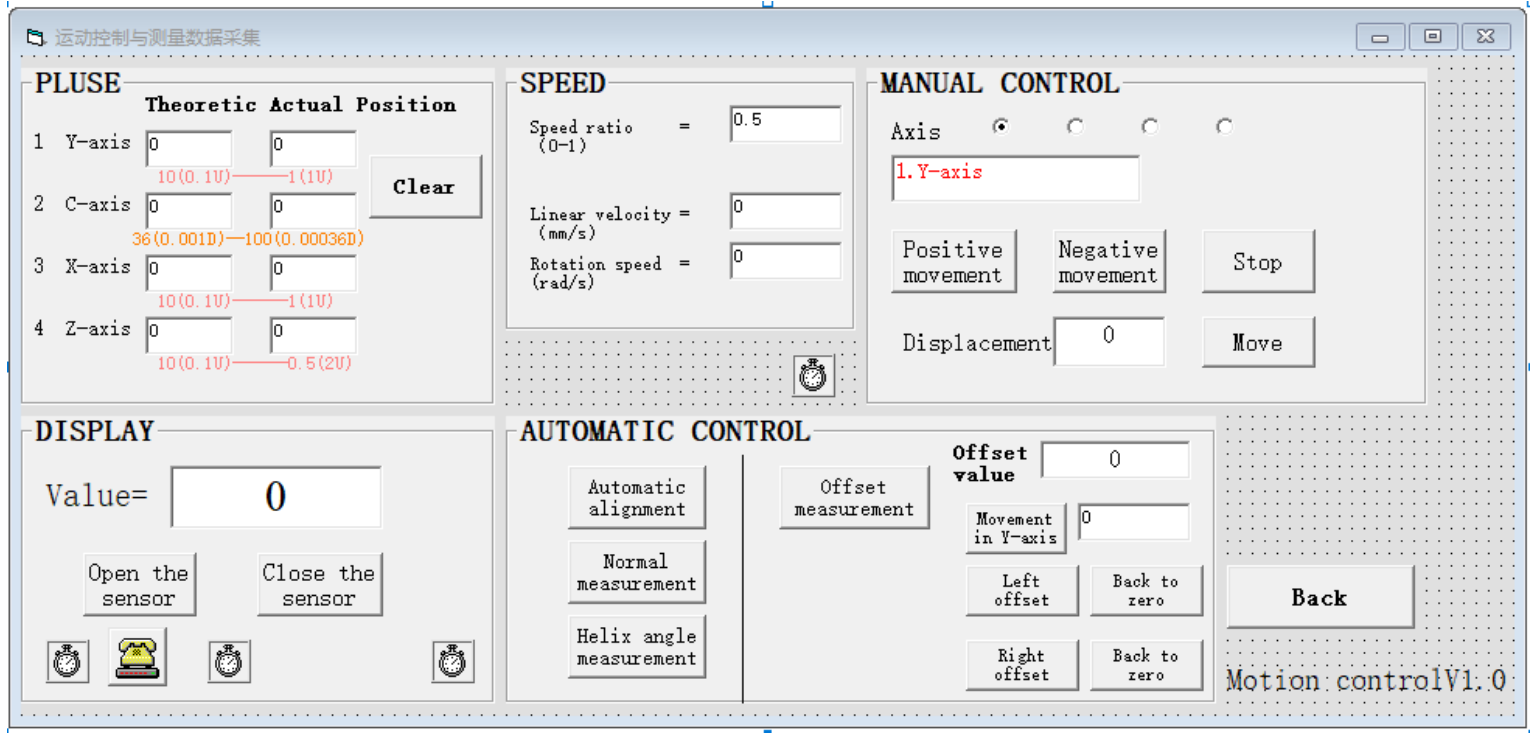

Fig. 9 The software system

The software system is shown in Fig. 9. The coordinate translation module is used to control the movement of the probe in the three directions of $\mathrm{XYZ}$ and the rotation of the workpiece turntable in the $\mathrm{C}$ axis direction. The speed control module is used to control the movement speed of the four axes, 
and the grating feedback module feedback the theoretical displacement and actual displacement of each axis, the measured value display module displays the current laser displacement sensor readings, the automatic control module can realize automatic alignment of shaft center, and the offset measurement module can realize automatic offset measurement. The spatial position of the laser probe or worktable can be adjusted by manual control, or automatic control, tooth profile measurement and offset measurement can be performed by automatic control.

\section{Experiment}

\subsection{Experimental device}

In offset measurement, the relative spatial position of the laser beam and the gear is calibrated by the cylindrical shaft, that is, the laser beam passes through the center of the workpiece coordinate system when the reading of the laser displacement sensor moves along the $\mathrm{X}$-axis guide rail reaches the maximum value. Then according to the offset value e and gear parameters, move the laser displacement sensor along the $\mathrm{X}$-axis rail to the offset position, and along the $\mathrm{Y}$-axis rail to the measurement position, so that the gear reference circle is located at the range $\mathrm{D}$ of the sensor.

The offset measurement experiment is shown in Fig. 10. The gear is installed on the shaft, and the sensor is offset by a certain distance from the gear center. During measurement, the gear rotates at a constant speed ratio and the encoder on the workpiece turntable records the angular pulse data of the measured point on the gear. At the same time, the laser displacement sensor collects the displacement data of the measured point relative to the laser source. The angular displacement data is output to the file through the measurement software.

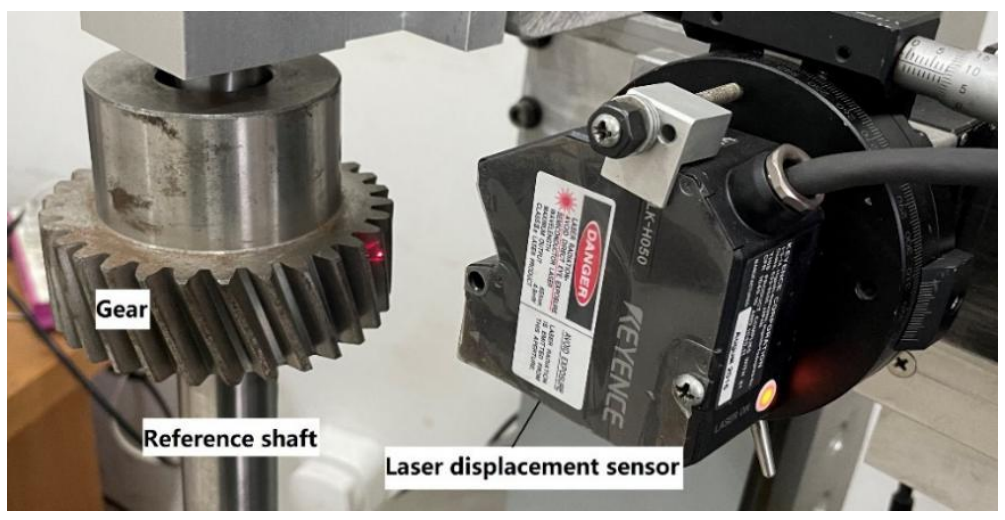

Fig.10 Gear offset measurement experiment 


\subsection{Data conversion}

Take any measurement result curve of a helical cylindrical gear with a number of teeth of 30 , a normal modulus of 3 , and a helix angle of $20^{\circ}$ as an example for data conversion. The measured offset is 23. As shown in Figure 11, the red line in the figure is the result of the measurement data, and the black line is the result of transforming the red line. The abscissa is the number of pulses of the measurement system, the workpiece rotates $360^{\circ}$ for 1 million pulses, and the ordinate is the distance from the measurement data point to the center of the gear. It can be seen from Fig. 11 that the black line is skewed before transformation, and the proportion of measured pulses at the tooth profile involute is significantly higher than the normal proportion. After coordinate transformation, the deflection is eliminated and the tooth profile conforms to the actual tooth profile.

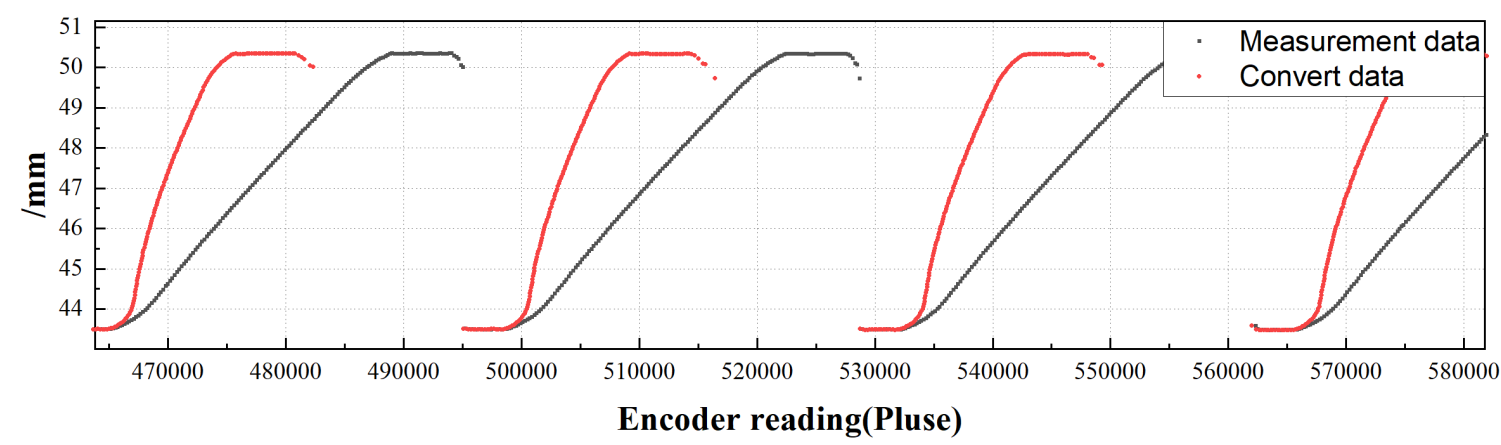

Fig.11 Coordinate conversion result with offset value 23

\subsection{Selection of the best offset value}

Taking a helical cylindrical gear with a number of teeth of 30 , a normal modulus of 3 , and a helix angle of $20^{\circ}$ as an example, 14 sets of different offset control tests are set according to the gear parameters, including $17,18,19,20,21,22,23,24,25,26,27,28,29,30$. Fig. 12 is a graph of the measurement data obtained with different offsets. Fig. 13 shows the measurement graphs in the three cases where the offset is 17,24 , and 30 . It can be seen that the offset is small, and the effective measurement points of the involute tooth profile are few. With the increase of the offset, the points of the involute tooth profile in the graph increase, the measurement graph becomes slower at the involute, the points at the dedendum decreases and the proportion in the measurement graph becomes smaller. As the offset continues to increase, the occlusion of the front teeth on the back teeth increases during 
the measurement process, and the dedendum is gradually blocked until it cannot be measured.

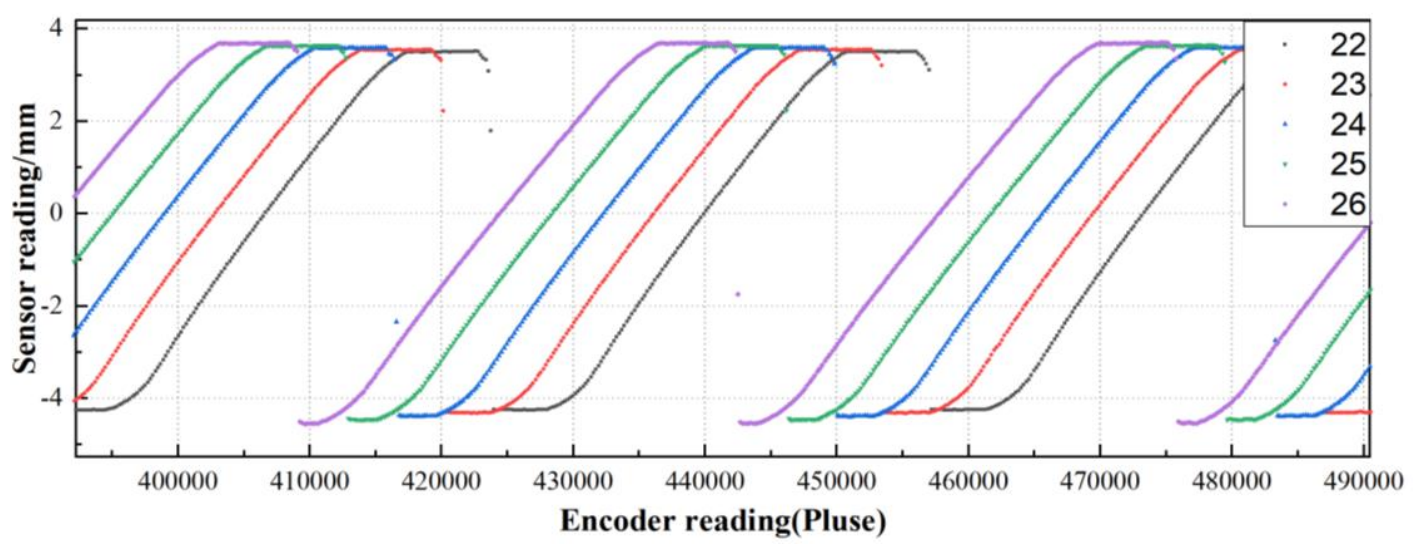

Fig.12 Measurement graphs for different offsets
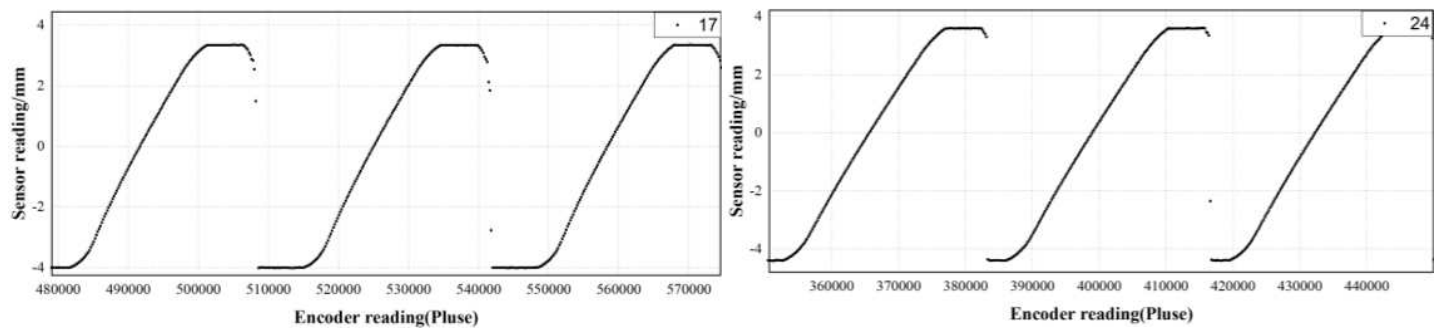

(a)Measurement graphs with offset value of 17 (b) Measurement graphs with offset value of 24

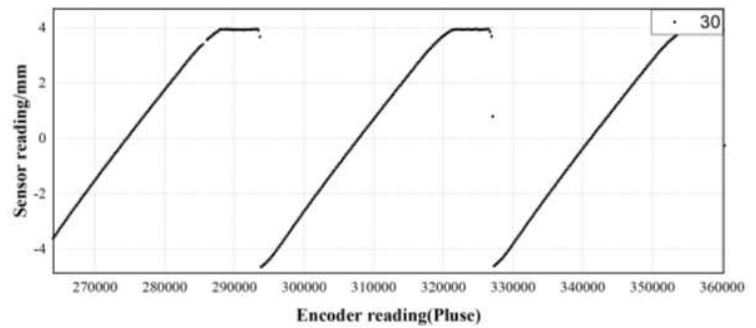

(c) Measurement graphs with offset value of 30

Fig.13 Measurement graphs at three different offsets

Among the offsets selected in the experiment, only the last group of the tooth profile is covered by the front teeth. The accuracy is judged by the number of valid data points. In the measurement data, the tooth profile part is counted from the dedendum radius measurements $+10 \%$ of the tooth height, and counts to the end of addendum radius measurements $-10 \%$ of the tooth height. As shown in Table 1, the selection of the offset value is positively correlated with the number of effective points of the tooth profile. Within a reasonable range of offset values, the larger the offset value is, the more the 
number of valid data points of the tooth profile. However, when the offset value is greater than 29 , the laser beam crosses the tooth tip and starts the measurement of the back tooth from the dedendum, the height difference increases the data fluctuation. At this time, the distance between the measurement starting point and the side of the tooth is relatively short, which affects the measurement of the tooth profile and shortens the effective measurement range.

Tab.1 Average number of effective data points for a single tooth under different offsets

\begin{tabular}{|c|c|c|c|c|c|c|c|c|c|c|c|c|c|c|}
\hline Offset value & 17 & 18 & 19 & 20 & 21 & 22 & 23 & 24 & 25 & 26 & 27 & 28 & 29 & 30 \\
\hline $\begin{array}{c}\text { Average number of valid data } \\
\text { points for a single tooth profile }\end{array}$ & 86 & 86 & 87 & 88 & 89 & 90 & 91 & 93 & 95 & 97 & 99 & 101 & 102 & 104 \\
\hline
\end{tabular}

Combine the diagram and the table. When the offset value is between 23-29, there are more effective data points and less data fluctuation, and when the offset value is 29 , the angle between the laser beam and the tooth profile normal is the smallest, and the effective data points are the most, which meets the conditions of the best offset value.

\subsection{Error}

The pitch deviation analysis and helix angle analysis are performed on the measured data with an offset of 23. In addition to one offset measurement, two measurements were taken on different sections of the gear to measure the helix angle.

(a)Pitch deviation analysis result

When analyzing the tooth pitch deviation, first carry out the coordinate conversion of the measured data, connect the data points through the spline curve, and use the dichotomy iteratively to find the intersection point of the reference circle and the spline curve. The distance between adjacent intersections is an individual pitch deviation, the difference between the individual pitch and the nominal pitch is a single pitch deviation, and the difference between the actual arc length and the nominal arc length of $\mathrm{k}$ pitches is the cumulative pitch deviation. Tab. 2 shows the individual pitch deviations and cumulative pitch deviations of some teeth. Fig. 13 shows the individual pitch deviation graphs of all 30 teeth of the tested gear. Fig. 14 shows the cumulative pitch deviation graphs. The maximum pitch deviation of this gear is: $0.02155 \mathrm{~mm}$, the minimum pitch deviation is: $0.00269 \mathrm{~mm}$, 
and the maximum cumulative pitch deviation is $0.02977 \mathrm{~mm}$.

Tab.2 Pitch deviation with an offset value of 23

\begin{tabular}{|l|l|l|l|}
\hline Number & Pitch & Individual pitch deviation & Cumulative pitch deviation \\
\hline$\ldots$ & $\ldots$ & $\ldots$ & $\ldots$ \\
\hline 2 & 9.96928 & 0.02091 & 0.02091 \\
\hline 3 & 9.93671 & -0.01167 & 0.00923 \\
\hline 4 & 9.95896 & 0.01058 & 0.01982 \\
\hline 5 & 9.96029 & 0.01191 & 0.03173 \\
\hline 6 & 9.93681 & -0.01157 & 0.02016 \\
\hline$\cdots$ & $\ldots$ & $\ldots$ & $\ldots$ \\
\hline
\end{tabular}

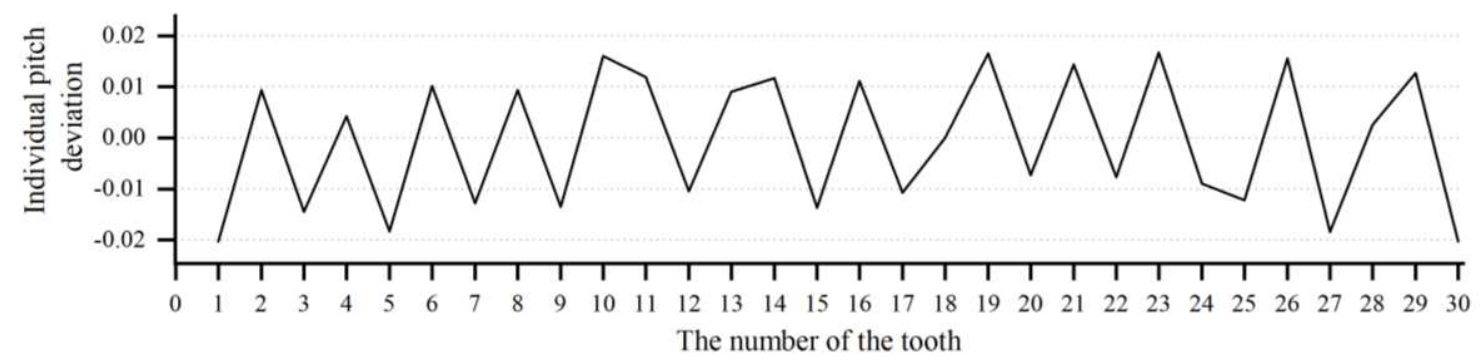

Fig.13 Individual pitch deviation

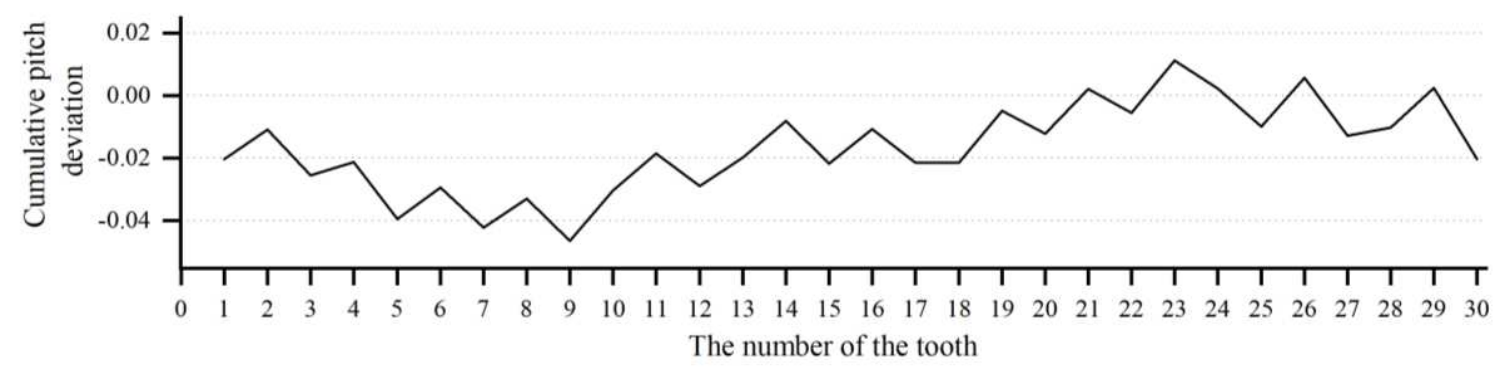

Fig.14 Cumulative pitch deviation

(b)Helical angle measurement result

After converting the measurement data on the different sections of the gear, calculate the intersection point of each set of data with the reference circle. Fit the straight line equation to three points on the same tooth, determine the slope $\mathrm{k}$ value of the straight line equation by the least square method, and finally get the helix angle. Table 3 lists the calculated helix angles of some teeth, and the actual helix angle of the gear is $20.5739^{\circ}$. 
Tab.3 Analysis of helix angle with offset value of 23

\begin{tabular}{|c|c|c|c|c|c|c|c|c|c|}
\hline Number & $\cdots$ & 15 & 16 & 17 & 18 & 19 & 20 & 21 & $\cdots$ \\
\hline Helix angle & $\cdots$ & 20.55892 & 20.86931 & 20.85579 & 20.95526 & 21.06316 & 20.86795 & 20.94725 & $\cdots$ \\
\hline
\end{tabular}

Experiments show that this measurement method is suitable for rapid and precise measurement of gears, provides a new idea for the laser measurement method of gears, and is important to gear measurement.

\section{8. conclusion}

(1) This paper proposes an offset laser measurement method for the deviation analysis of cylindrical gear. By offsetting the laser beam and the gear for a certain distance, the accuracy and stability problems caused by object surface inclination error. we provide the basis for the selection of the offset by discussing the optimal offset model, and proposes a coordinate conversion method to process the offset measurement data. The feasibility of the method is verified by experiments.

(2) The selection of offset is affected by gear parameters, the front tooth occlusion degree, the slop of the tooth profile normal line and overall data efficiency. According to the gear parameters, the minimum offset determined by the slop of the tooth profile normal line, and the maximum offset determined to ensure the efficiency of the data. Measure at the maximum value in the interval, the angle between the laser beam and the tooth profile normal is the smallest, and the effective data points are the most, which meets the conditions of the best offset value.

(3) The offset laser measurement method proposed in this paper optimizes the angle of laser beam and the normal vector of the tooth profile. Compared with the laser measurement through the gear center, the measurement can collect more data points of the tooth profile, which can analyze multiple errors, improve the measurement accuracy and data stability, and is suitable for precision measurement of gears. It can be used to measure other types of gears or rotating bodies.

\section{Declarations}

\section{Funding}

This work was supported by the National Natural Science Foundation of China [grant number $51475409]$. 


\section{Conflicts of interest/Competing interests}

No known competing financial interests or personal relationships that could have appeared to influence the work reported in this paper.

\section{Availability of data and material}

Not applicable.

\section{Code availability}

Not applicable.

\section{Ethics approval}

Not applicable.

\section{Consent to participate}

Not applicable.

\section{Consent for publication}

Not applicable.

\section{References}

[1] Zhang Zhan, Wen Chengzhen, Zeng Jianfeng. Gear detection technology [M]. China Machine Press, 2012.1.

[2] Jin Jiaqi, Li Wenlong, Duan Zhenyun. Principle and technology of large gear on machine measurement [M]. China Machine Press:,2016.3.

[3] MA DongHui,WUJiaNing,LIUTao,YANShaoZe.Deformation analysis of the flexspline of harmonic drive gears considering the driving speed effect using laser sensors[J].Science China(Technological Sciences), 2017, 60(08): 1175-1187.

[4] Tian Hao, WuFan, GongYongjun. Gear Tooth Profile Reconstruction via Geometrically Compensated Laser Triangulation Measurements.[J]. Sensors (Basel, Switzerland),2019,19(7).

[5] Naresh K. Raghuwanshi,AnandParey. Experimental Measurement of mesh stiffness by laser displacement sensor technique[J]. Measurement,2018.

[6] HanjunJiang,XiaojieSun,Fuhao Liu. Measurement method of tooth friction force for helical gears by laser displacement sensor[J]. Journal of the Brazilian Society of Mechanical Sciences and Engineering,2020,42(3-5).

[7] Qiu Lin.Research and Development of Gear Laser Precision Measuring Device and Software System[D]. Yangzhou 
university,2017.

[8] Jakub Sandak, ChiakiTanaka,TadashiOhtani. Evaluation of surface smoothness by a laser displacement sensor II: comparison of lateral effect photodiode and multielement array[J]. Journal of Wood Science,2004,50(1).

[9] Sun Bin, Li Bing.A quantitative error compensation model of the inclination angle of the laser displacement sensor[J]. Chinese Journal of Scientific Instrument,2015,36(05):996-1004.

[10] Yong-Chen Pei,Hai-Liang Xie,Qing-Chang Tan. A non-contact high precision measuring method for the radial runout of cylindrical gear tooth profile[J]. Mechanical Systems and Signal Processing,2020,138.

[11] Huang Xiaopin. Accuracy analysis and uncertainty evaluation of laser sensor displacement measurement[D].Dalian University of Technology,2012. 
Figures

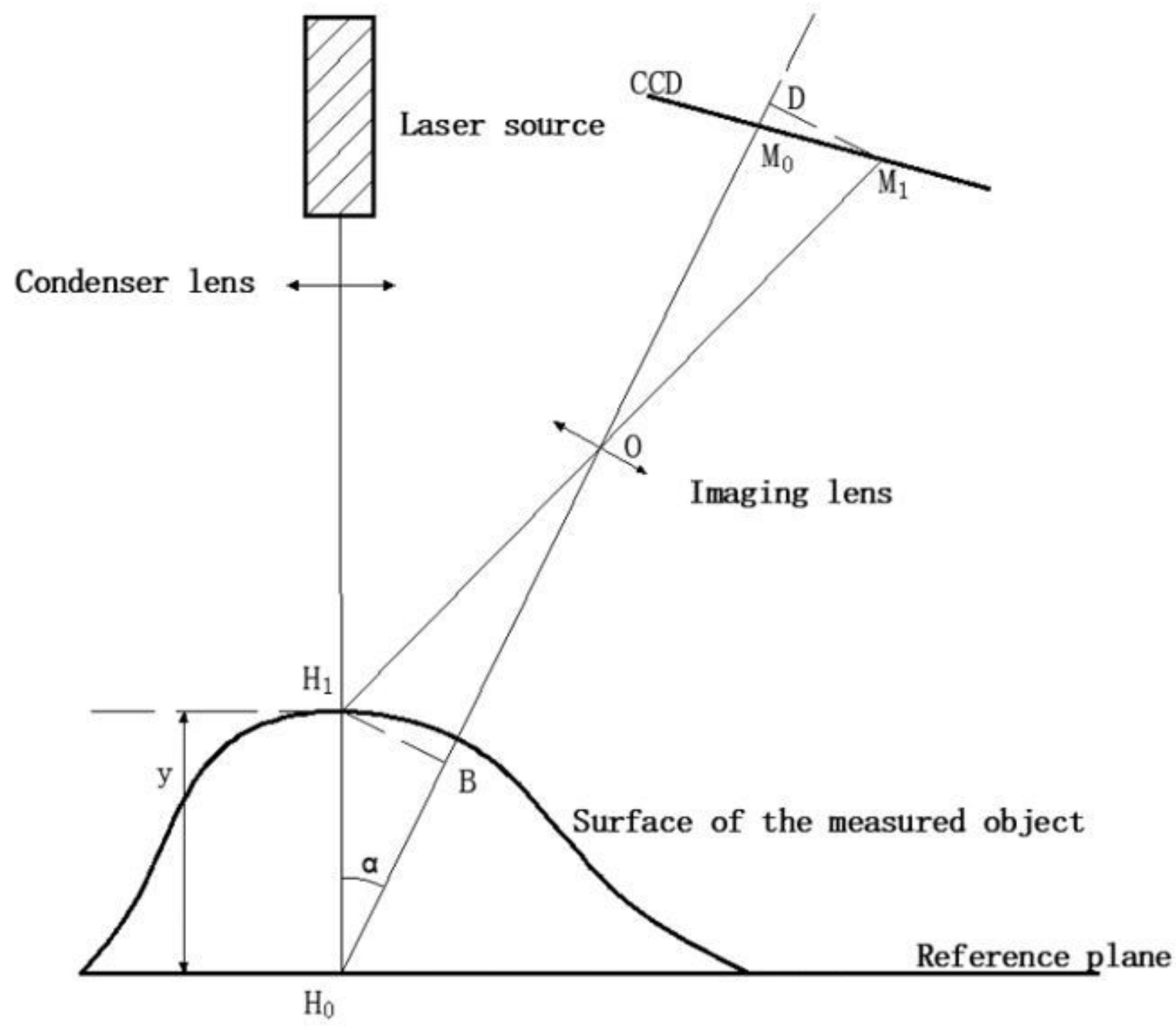

Figure 1

The principle of triangulation 


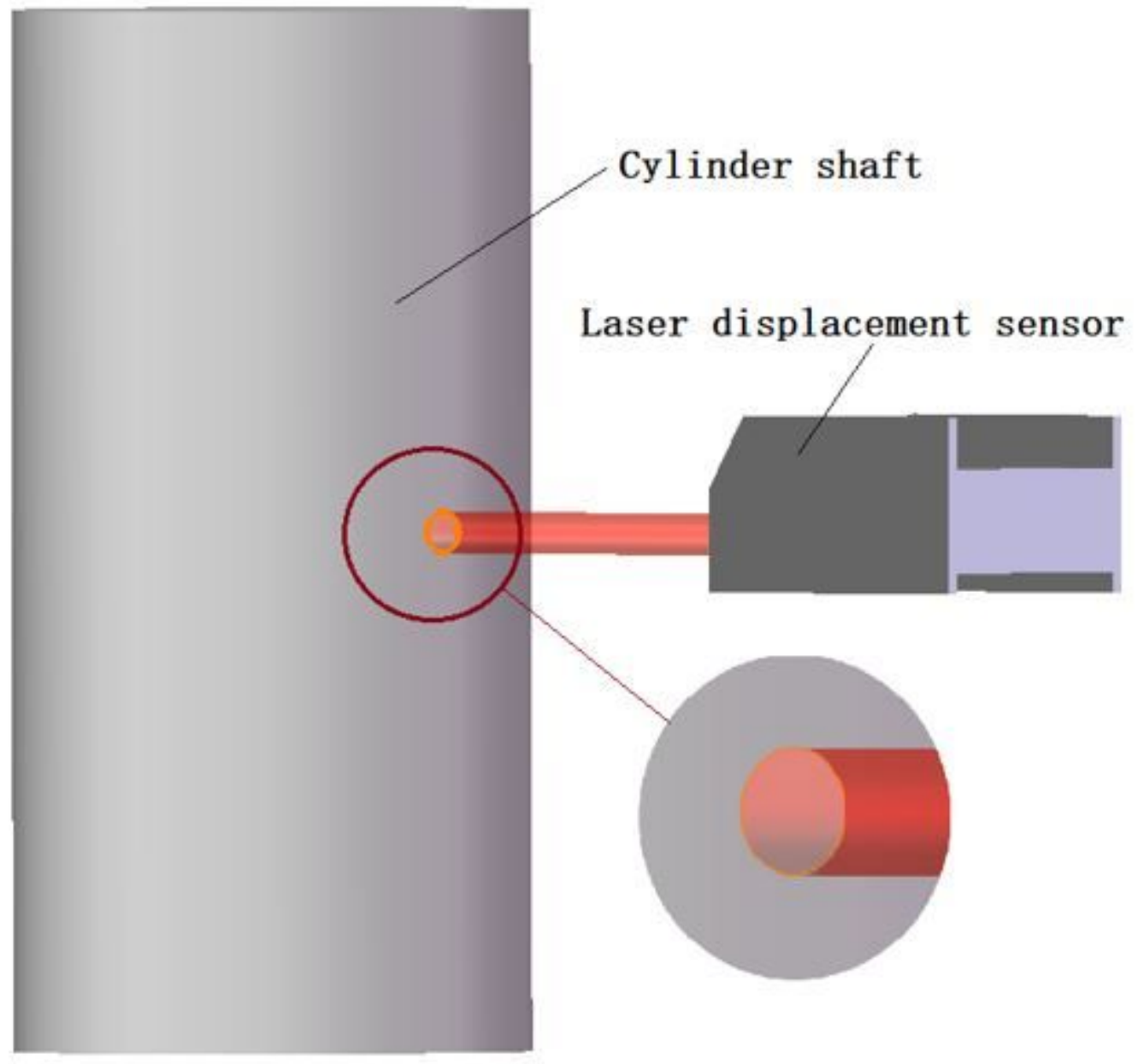

Figure 2

The spot shape when laser beam facing the center of the shaft 


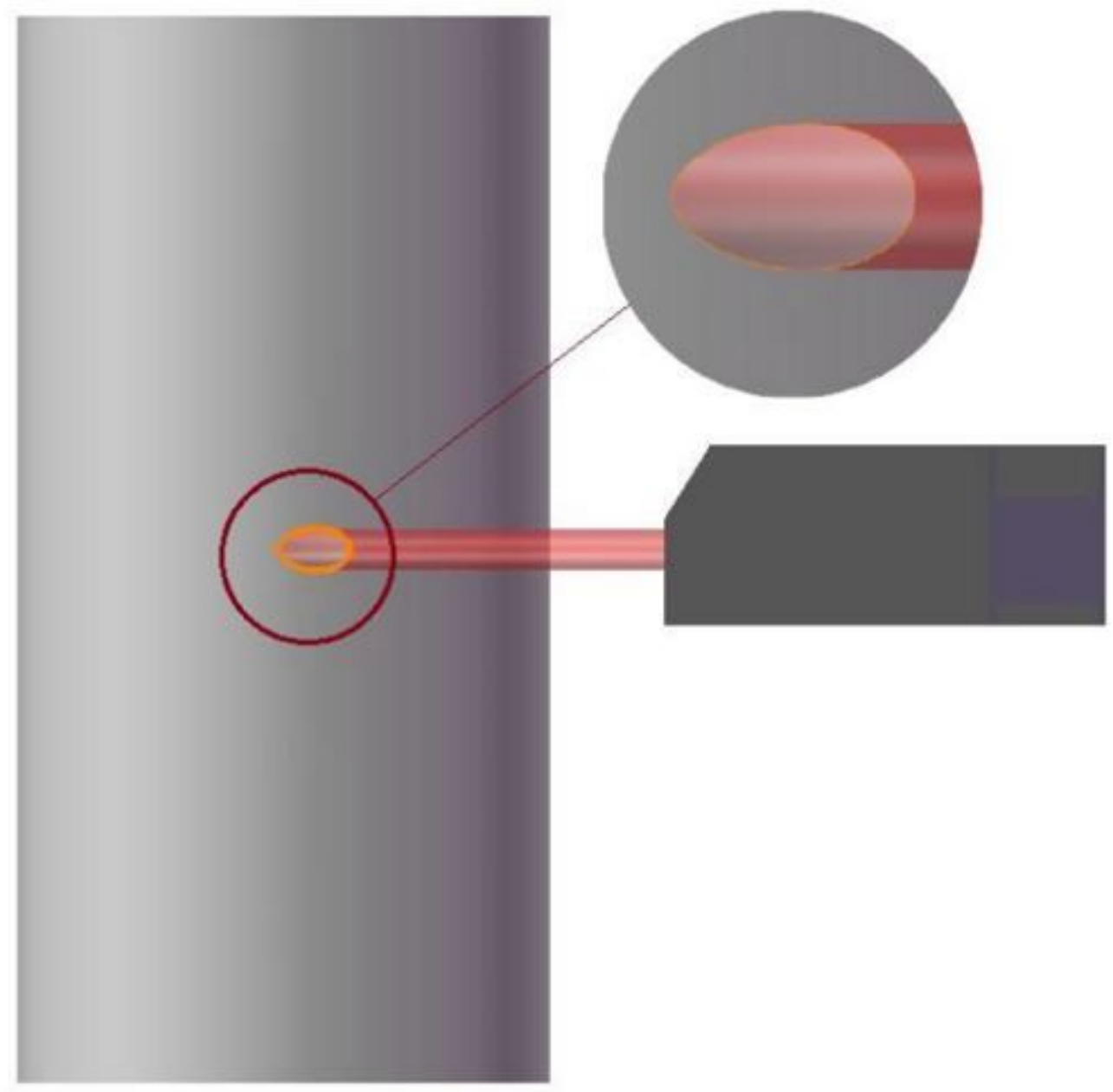

Figure 3

The spot shape when laser beamoff-center 


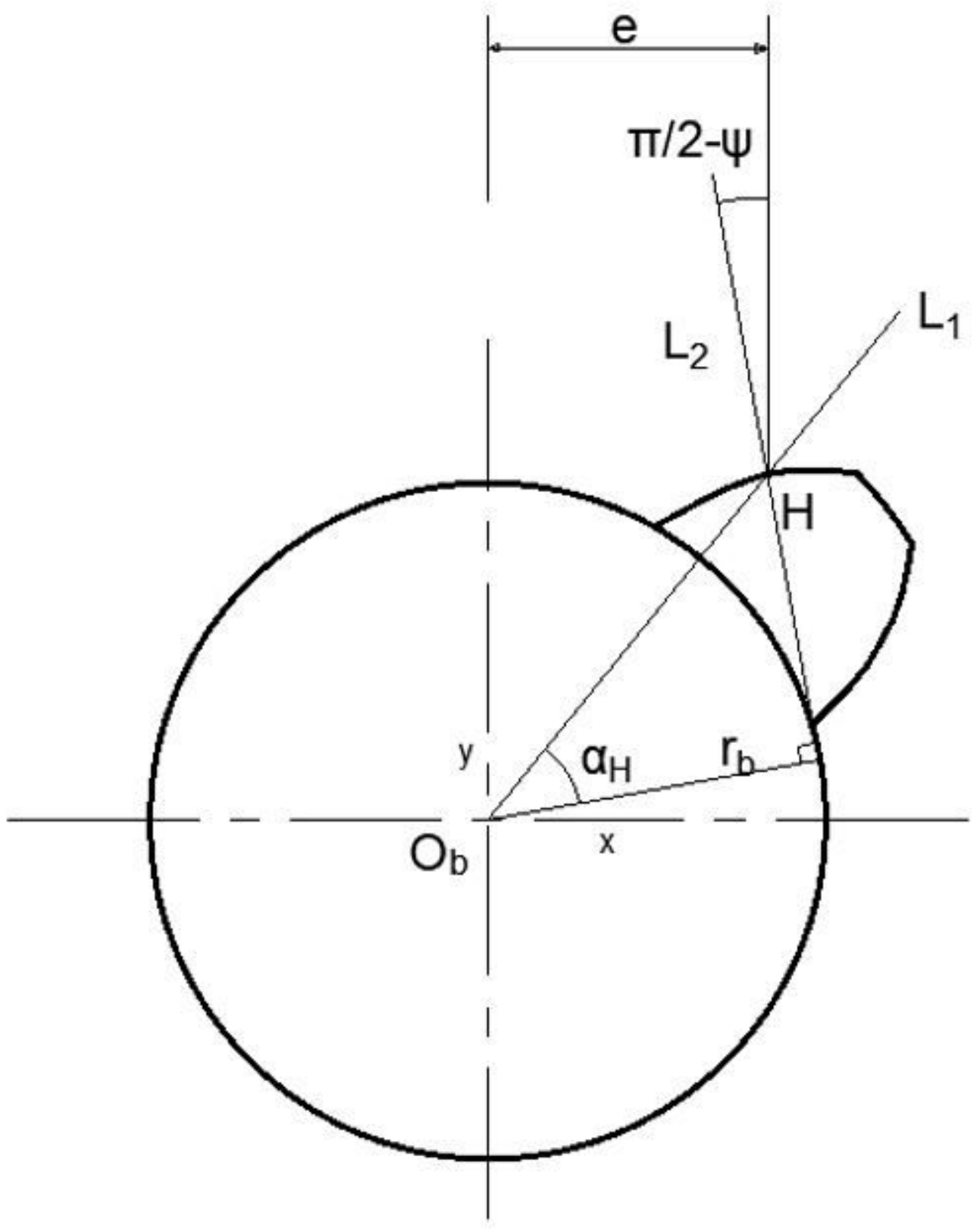

Figure 4

Schematic diagram of the normal of the tooth profile 

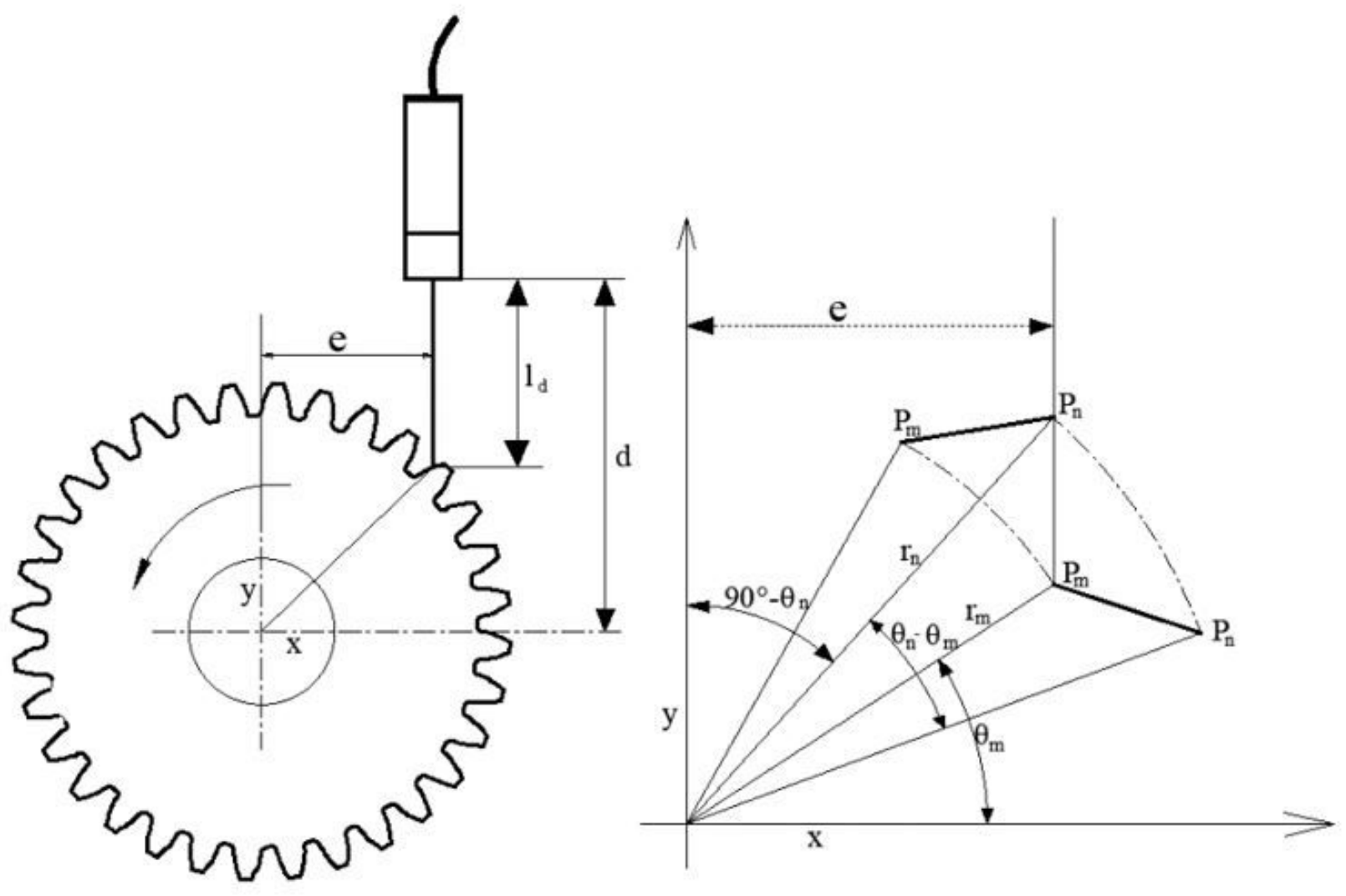

Figure 5

Schematic diagram of offset laser measurement method 


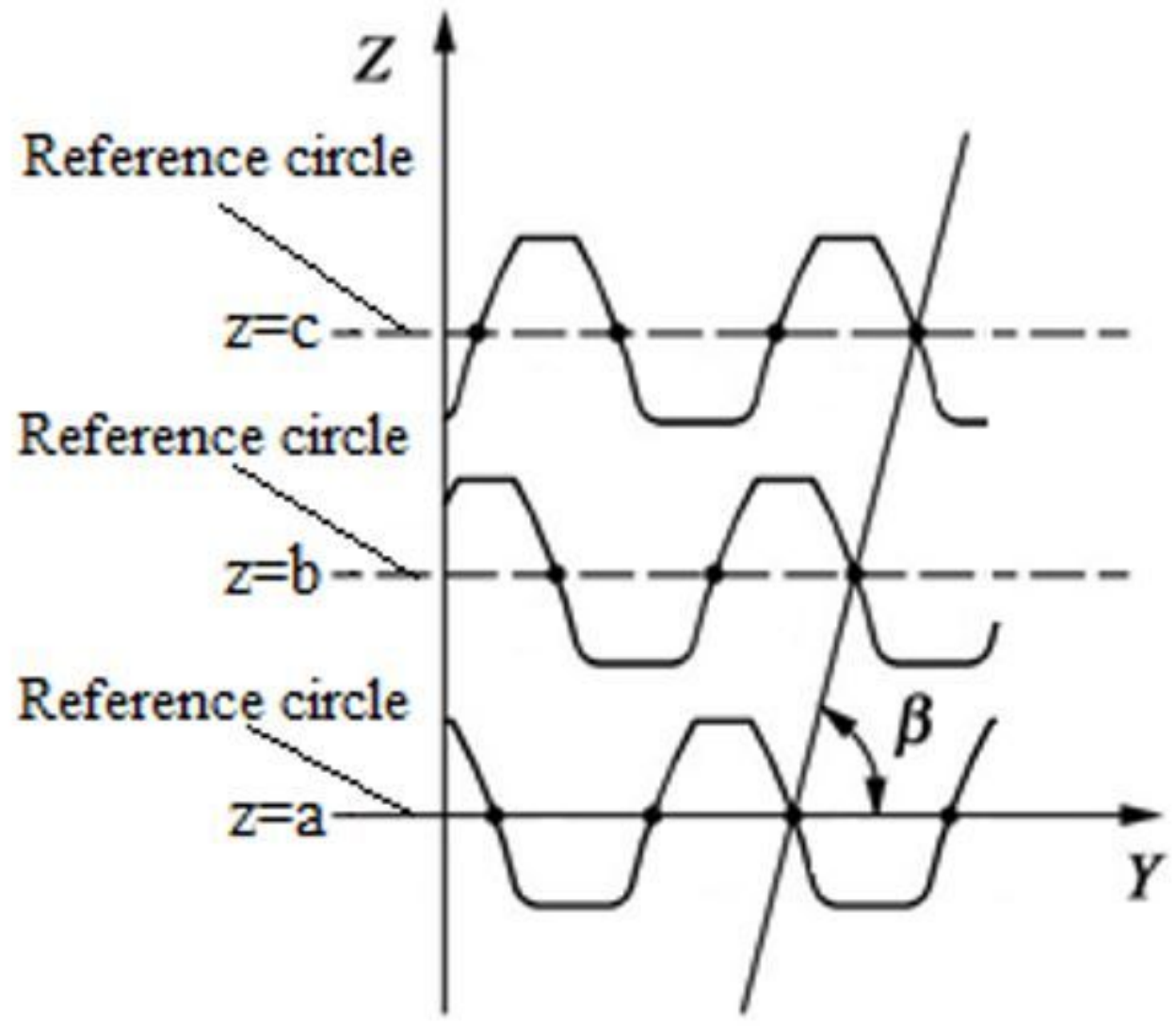

Figure 6

Schematic diagram of helix angle analysis 


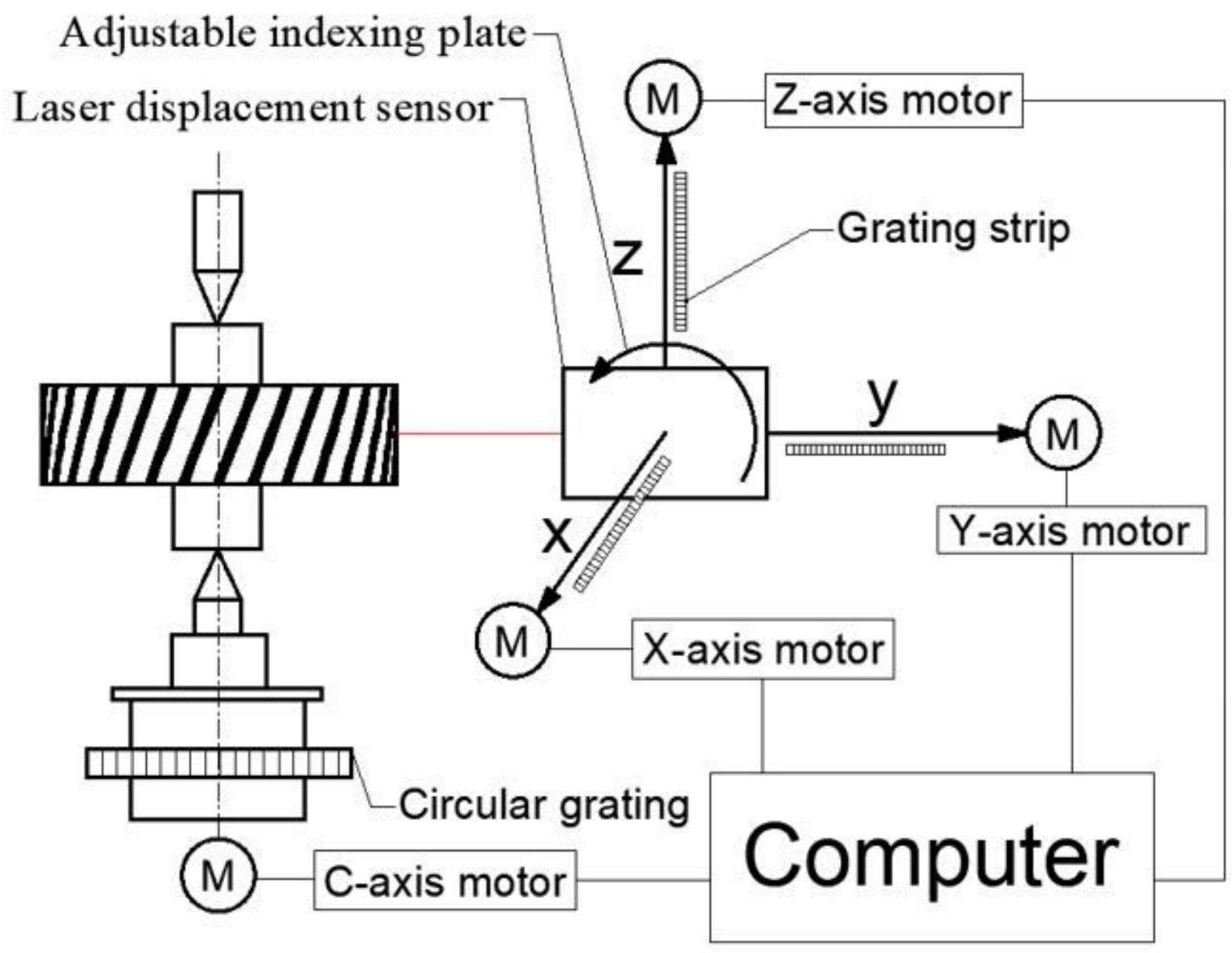

Figure 7

Measurement system 


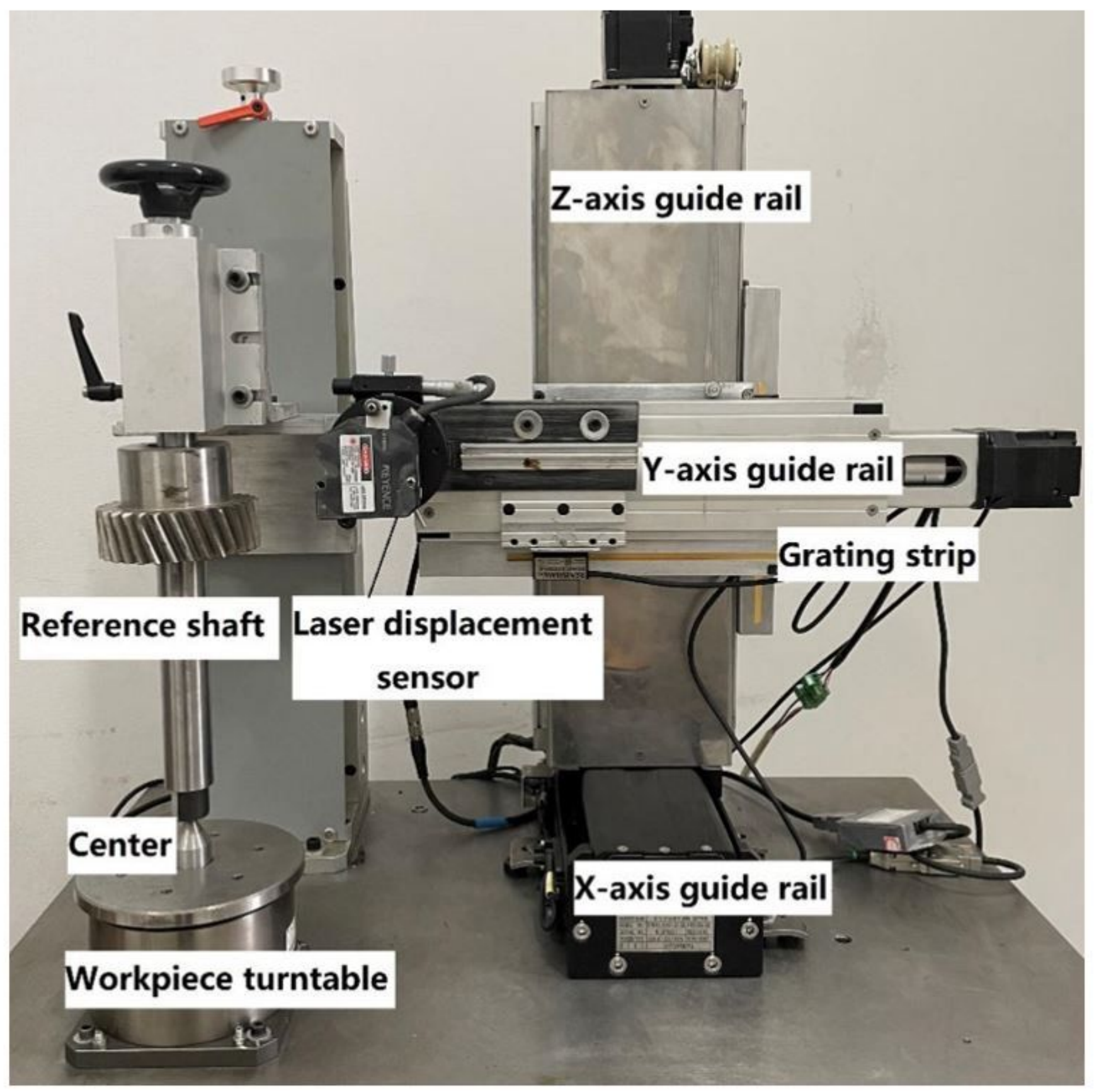

Figure 8

The measurement device 


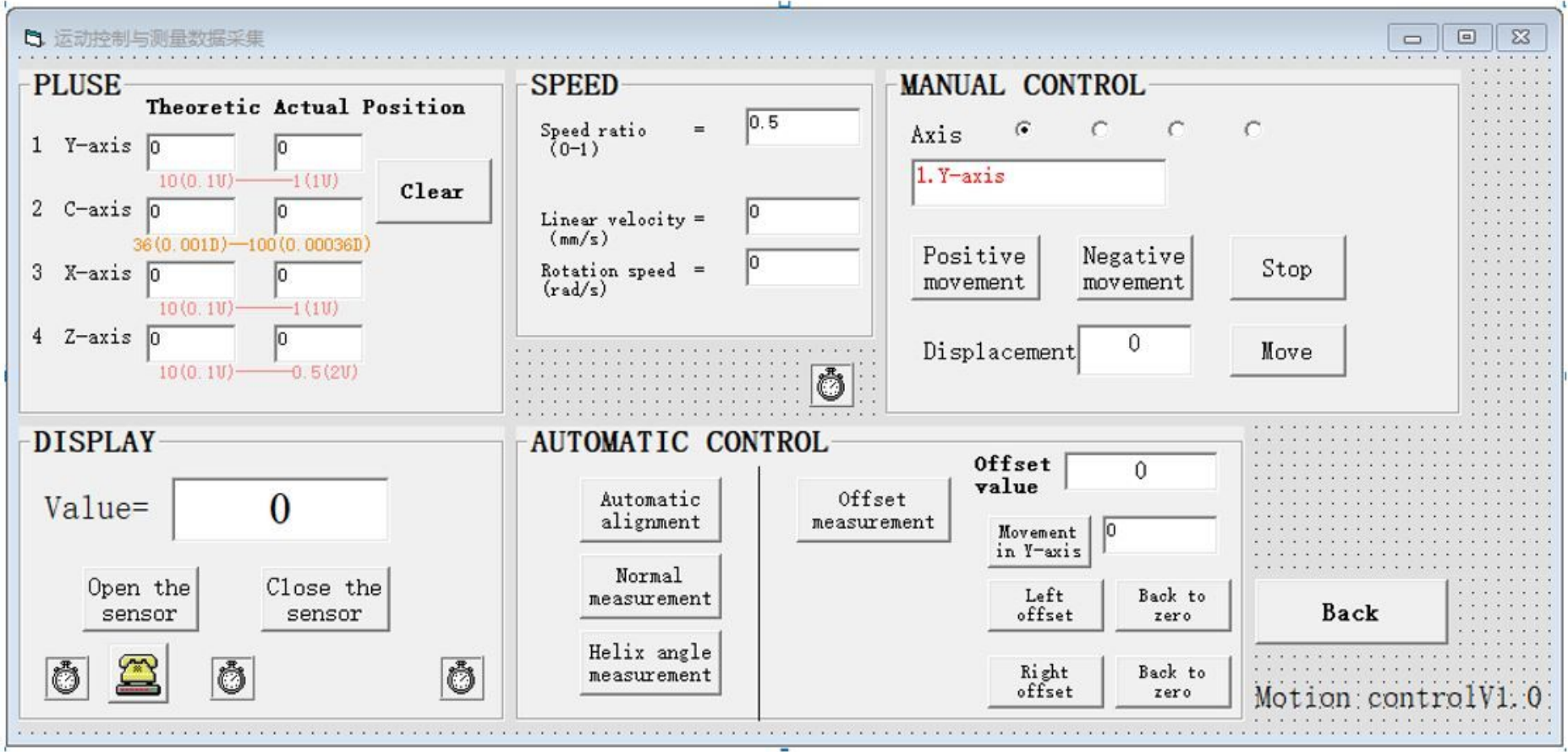

\section{Figure 9}

The software system

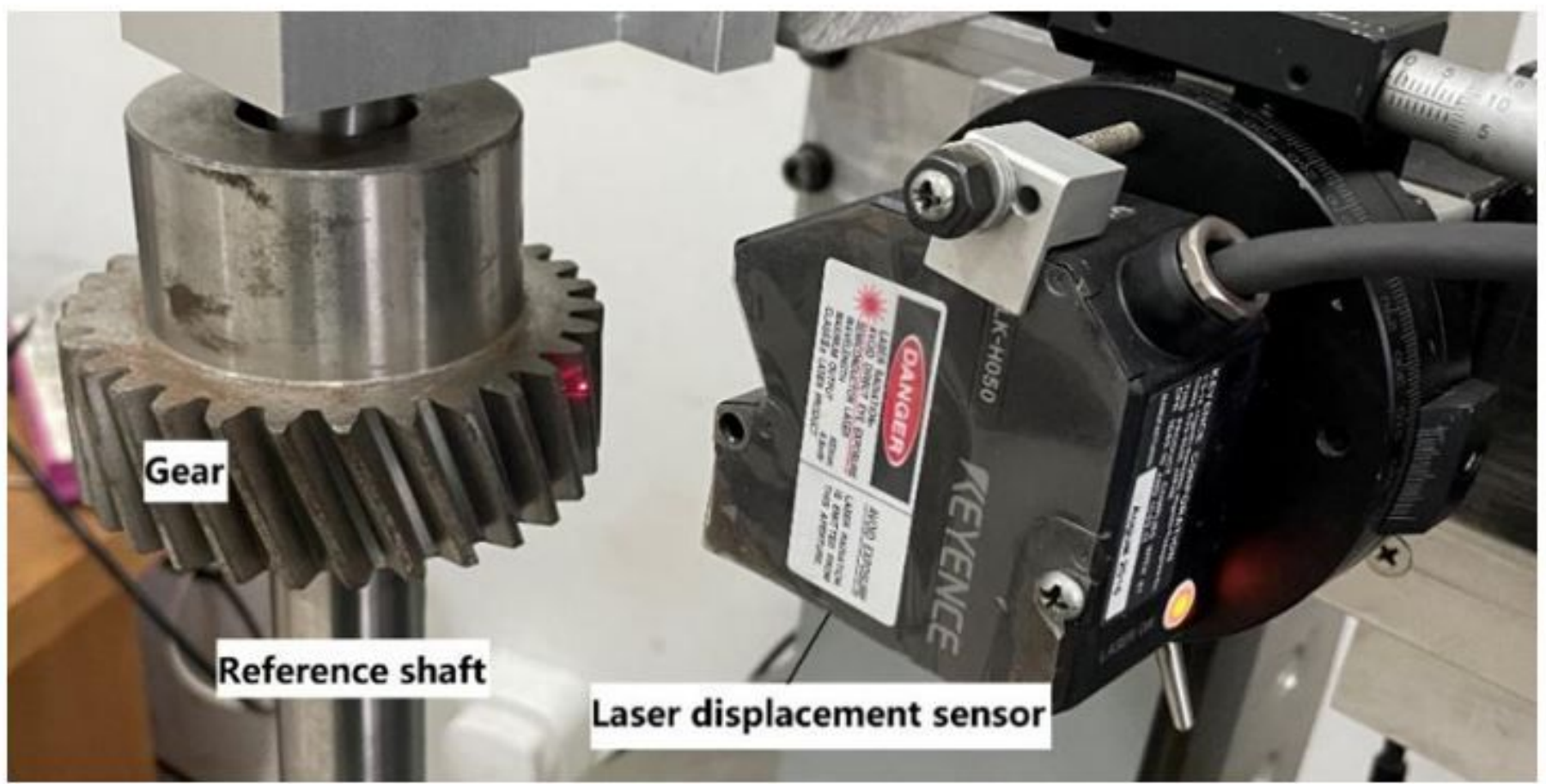

Figure 10

Gear offset measurement experiment 


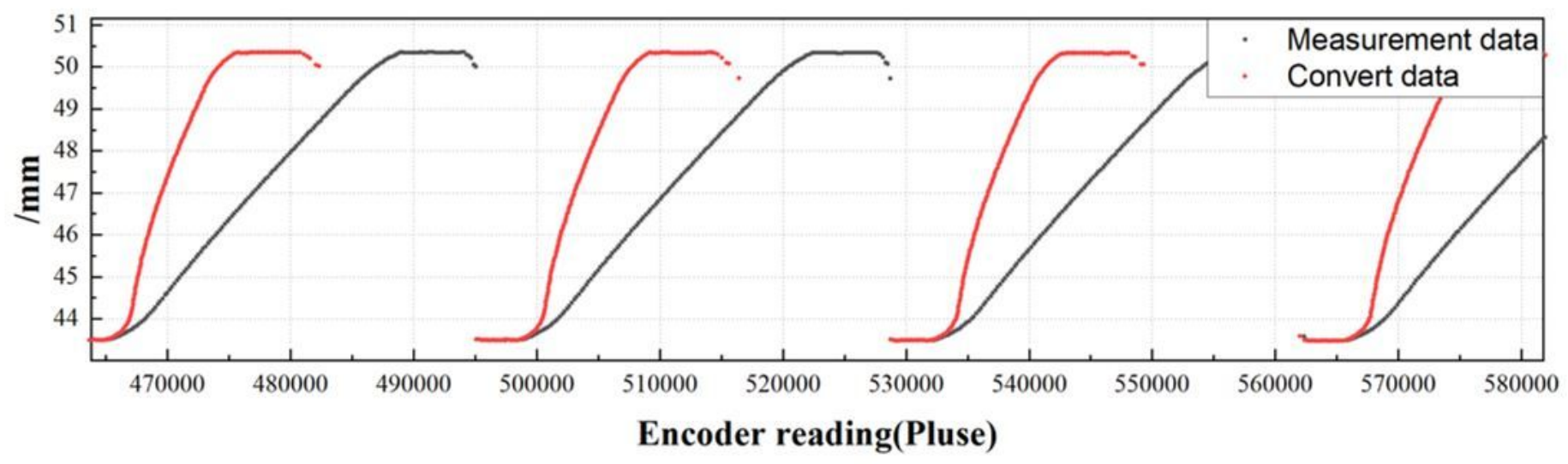

Figure 11

Coordinate conversion result with offset value 23

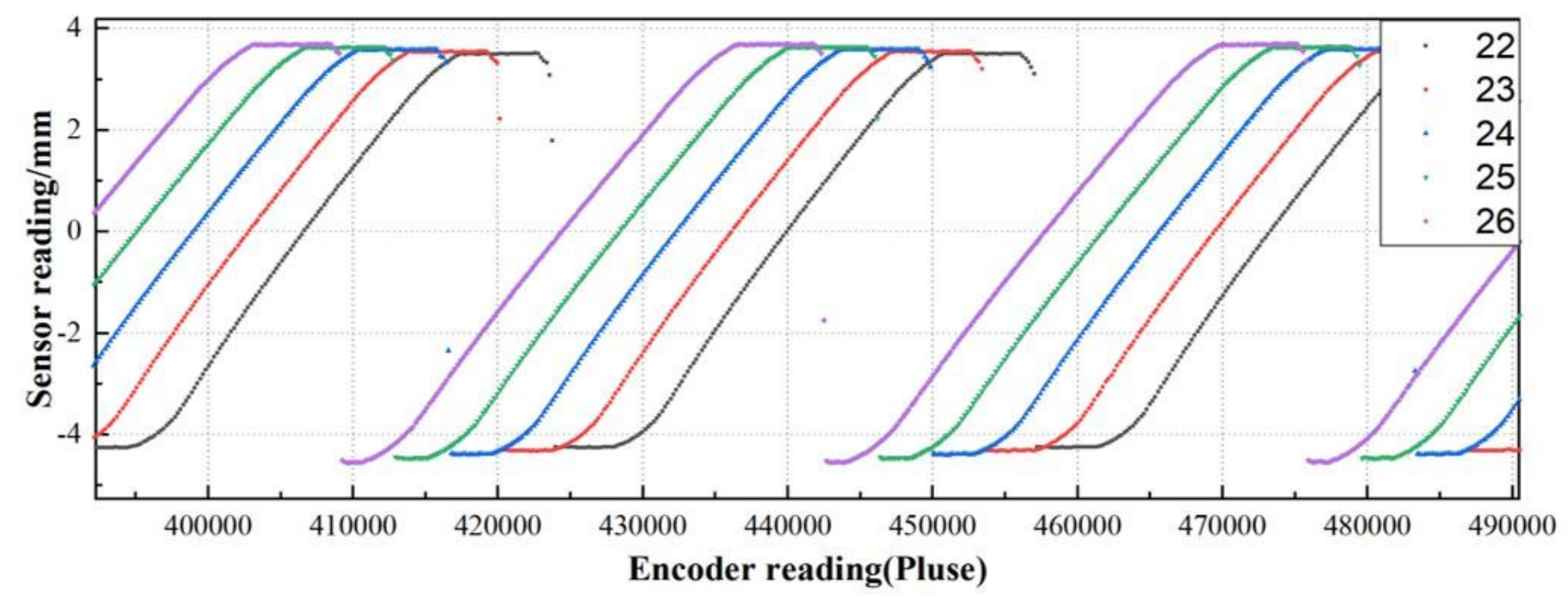

Figure 12

Measurement graphs for different offsets 

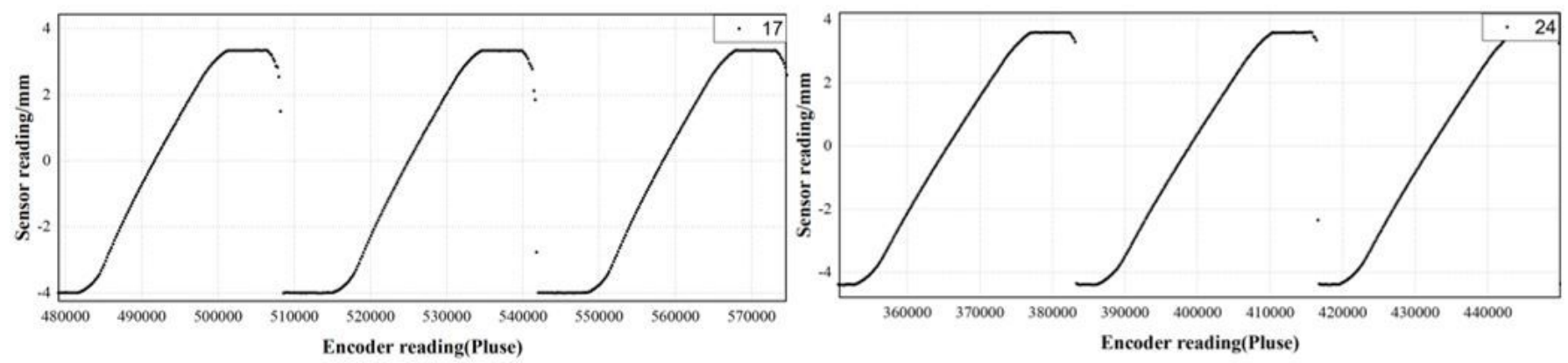

(a)Measurement graphs with offset value of 17 (b) Measurement graphs with offset value of 24

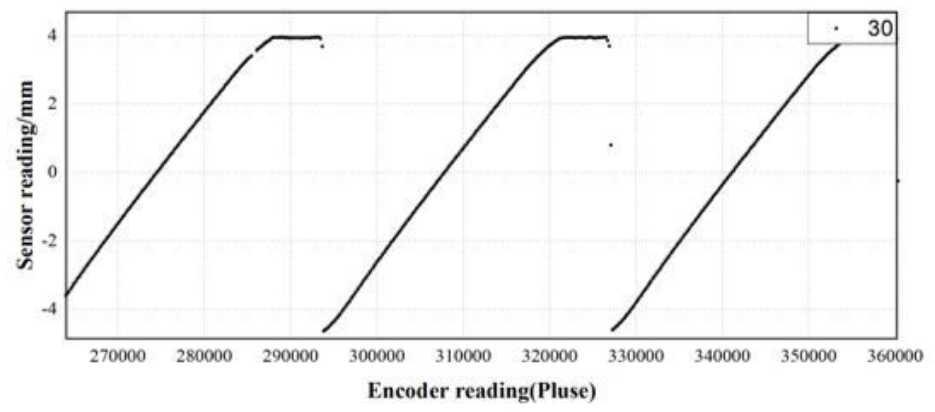

(c) Measurement graphs with offset value of 30

\section{Figure 13}

Measurement graphs at three different offsets

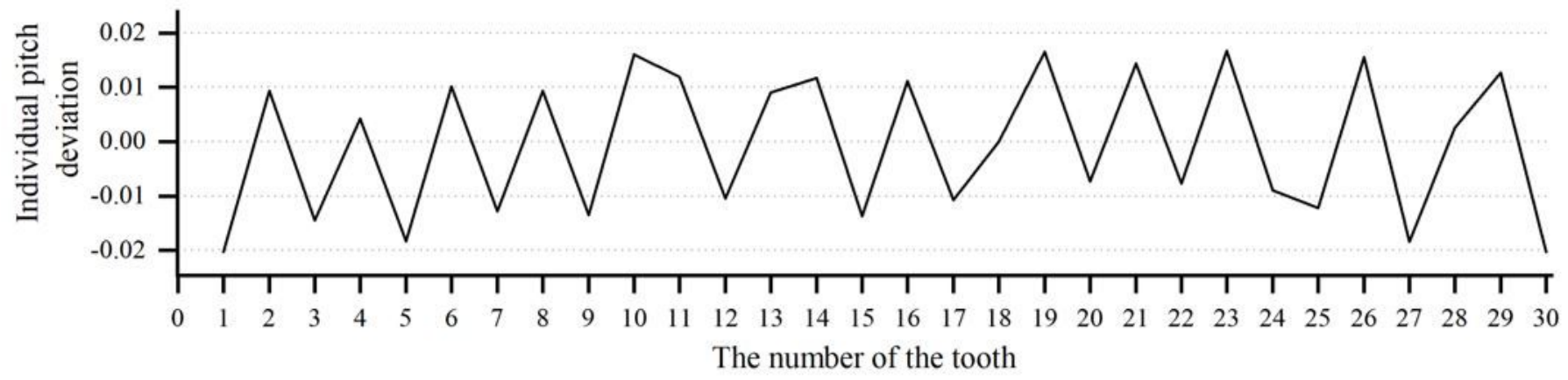

Figure 14

Individual pitch deviation 


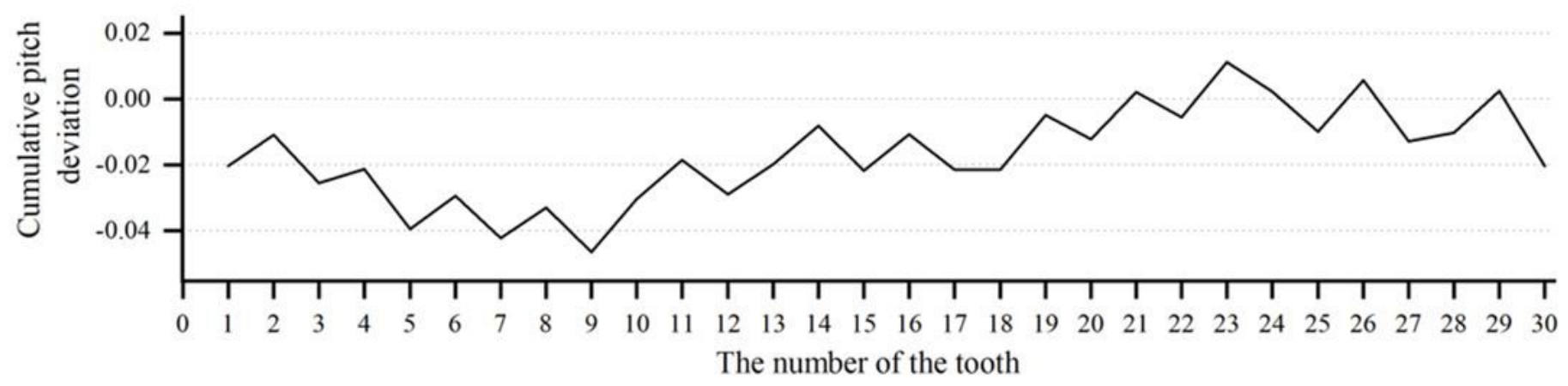

\section{Figure 15}

Cumulative pitch deviation 